Portland State University

PDXScholar

Civil and Environmental Engineering Master's

Project Reports

Spring 2016

\title{
Performance Implications of Bicycle Specific Treatments at Signalized Intersections
}

Andrew Kading

Portland State University

Follow this and additional works at: https://pdxscholar.library.pdx.edu/cengin_gradprojects

Part of the Civil Engineering Commons

Let us know how access to this document benefits you.

\section{Recommended Citation}

Kading, Andrew, "Performance Implications of Bicycle Specific Treatments at Signalized Intersections" (2016). Civil and Environmental Engineering Master's Project Reports. 27.

https://doi.org/10.15760/CEEMP.8

This Project is brought to you for free and open access. It has been accepted for inclusion in Civil and Environmental Engineering Master's Project Reports by an authorized administrator of PDXScholar. Please contact us if we can make this document more accessible: pdxscholar@pdx.edu. 
Performance Implications of Bicycle Specific Treatments at Signalized Intersections.

BY

\begin{abstract}
Andrew Kading
A research project report submitted in partial fulfillment of the requirement for the degree of

\section{MASTER OF SCIENCE \\ IN \\ CIVIL AND ENVIRONMENTAL ENGINEERING}

Project Advisor:

Dr. Christopher Monsere

Portland State University

(C)2016 


\section{ACKNOWLEDGMENTS}

The author would like to thank the following people and organizations with their help, feedback, and support: Dr. Sirsha Kothuri for her patience and guidance. Dan Brandesky at Econolite, who by any metric is a signal controller rock star. Dr. Monsere, for serving as the advisor. NITC for funding the research. Paul Ziebell from PBOT for the technical assistance and patience, as well as PBOT in general for much of the information needed to complete this work. I would also like to thank my spouse, because she put up with all this. And finally you the reader, because without you this work is meaningless. 


\begin{abstract}
A rise in cycling nationwide has brought an increased awareness of bicycle specific safety concerns, particularly right-hook crashes at signalized intersections. There are several common and emerging signal timing strategies that are can be used to improve the safety and efficiency of traffic signals for people on bicycles, including the Leading Bicycle Intervals (LBI), Split LBI, and Exclusive Bicycle Phasing (EBP). The leading and split bicycle intervals provide a head start for bicycles in advance of concurrent and conflicting motor vehicle movements, typically about 3-5 seconds. The EBP provides an interval only when bicycles are moving. To examine the performance and efficiency metrics under a range of bicycle and motor vehicle volumes, a Vissim microsimulation model was developed. Passenger cars, large trucks, bicycles, and pedestrians were modeled to understand the performance across and between the modes. User delay values were extracted from the model and compared to the base case. A total of 210 simulation runs were conducted, accounting for approximately 262.5 hours of simulation.
\end{abstract}

Results indicate a small vehicle delay increase of between $4 \%$ - 11\% [as delay in seconds per user] for the Split LBI, with thru traffic (which is unaffected by the Split LBI treatment) virtually unaffected at 1\% - 2\% delay increases; the LBI saw larger increases in vehicle delay from $11 \%$ $19 \%$; and large vehicle delay increases for the EBP (up to $26 \%$ for the eastbound approach), but also found an apparent bias in the coordination scheme that led to decreases for the westbound approach. Bicycle delay results showed more variation with the LBI treatment showing little impact, with results ranging from $-5 \%$ - 5\% changes in bicycle delay. Split LBI showed delay increases of 3\% - 11\% for the thru cyclists, but mixed results for the right turning cyclists, which showed delay results increasing as a function of bicycle traffic. Bicycle delay results for the EBP showed large increases from 100\% - 565\%. Pedestrians and left turning vehicles were all but unaffected by either the LBI or Split LBI. While the EBP did show changes in delay as high as $22 \%$ for pedestrians.

This research demonstrates that bicycle specific treatments do impact user delay at busy arterial intersections, although the magnitude of impact differs depending on the treatment used. The use of the Split LBI had little overall impact, suggesting it is an effective treatment if the intersection configuration is correct. The LBI had greater impact, and should be considered for intersections with the correct configuration. The EBP had large delay increases suggesting limited use, only when bicycle volumes are high enough to justify the delay. 


\section{TABLE OF CONTENTS}

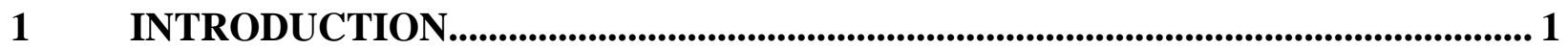

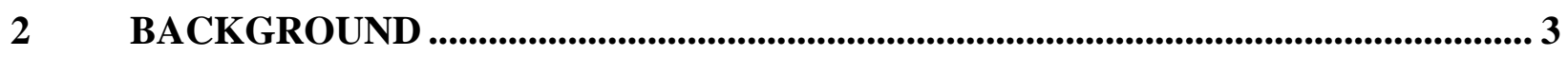

3 DEVELOPMENT OF SIMULATION MODEL …........................................................... 7

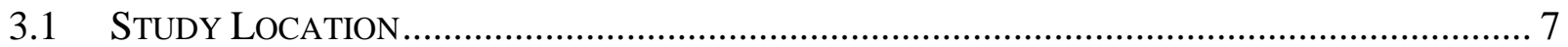

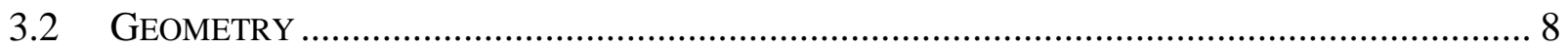

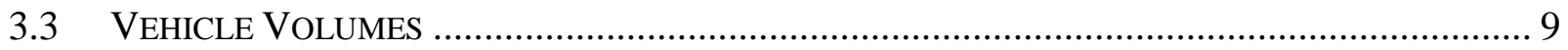

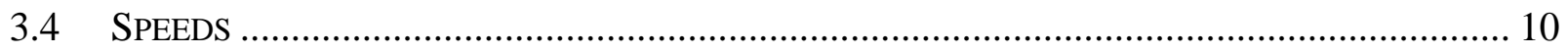

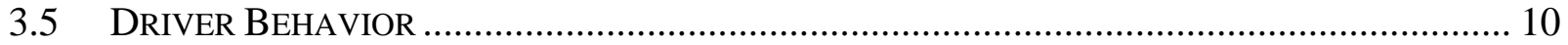

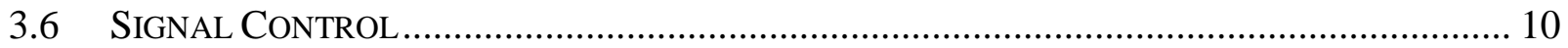

3.6.1 Leading Bicycle Interval and Split Leading Bicycle Interval .............................. 12

3.6.2 Exclusive Bicycle Phase .............................................................................. 16

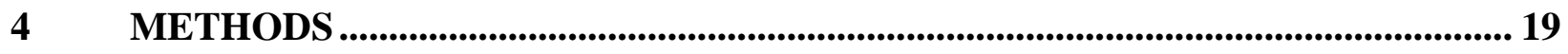

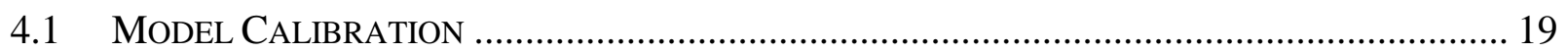

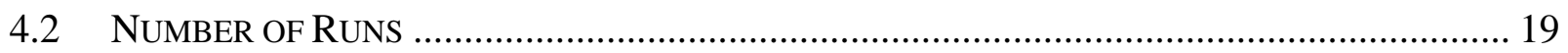

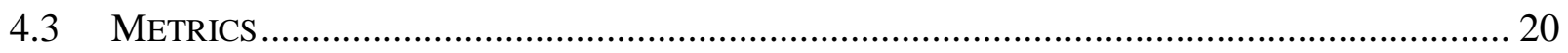

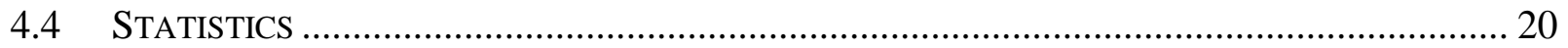

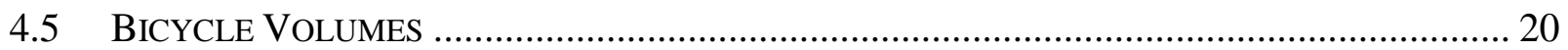

4.6 COORDINATED BASE CASE ............................................................................... 21

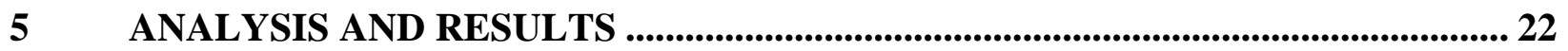

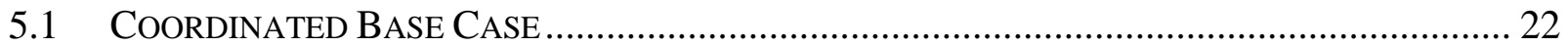

5.2 LEADING BICYCLE INTERVAL (LBI) ..................................................................... 22

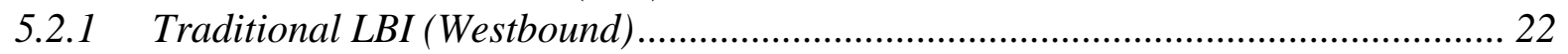

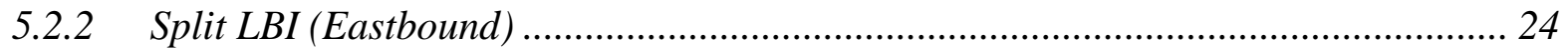

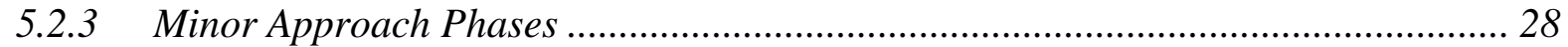

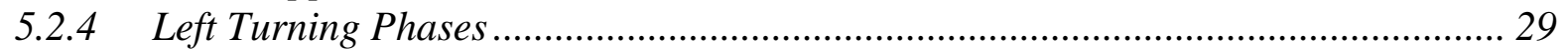

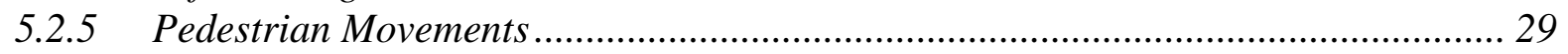

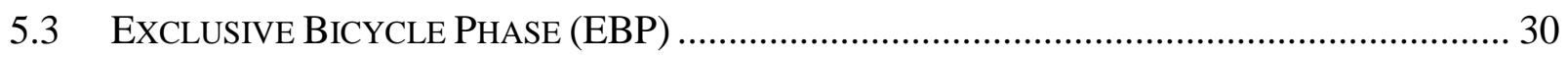

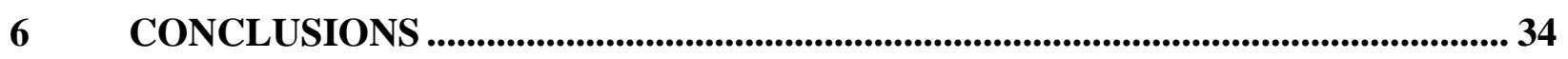

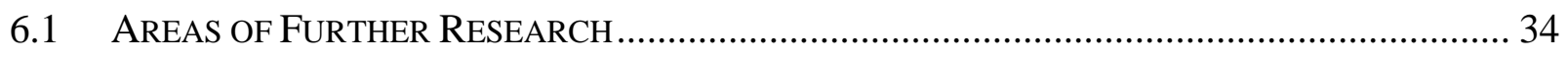

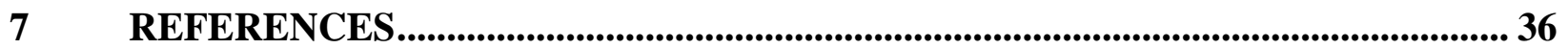




\section{LIST OF TABLES}

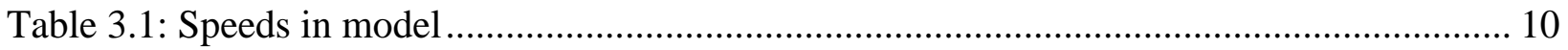

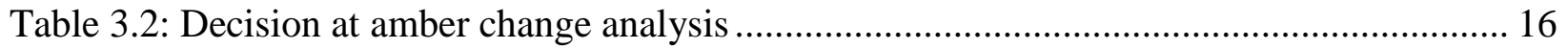

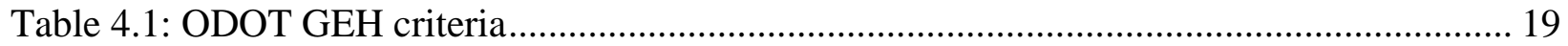

Table 4.2: Sensitivity analysis bicycle volumes .................................................................. 21

Table 5.1: LBI (Phase 6) Vehicle Sensitivity Analysis Delay Results ..................................... 24

Table 5.2: LBI (Phase 6) Bicycle Sensitivity Analysis Delay Results ..................................... 24

Table 5.3: Split LBI (Phase 2) Vehicle Sensitivity Analysis Delay Results ............................ 26

Table 5.4: Split LBI (Phase 2) Bicycle Sensitivity Analysis Delay Results .............................. 28

Table 5.5: Minor Phases (Phases 4 and 8) Vehicle and Bicycle Sensitivity Analysis Delay

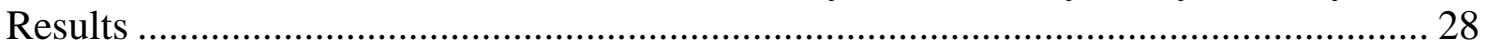

Table 5.6: Left Turn Vehicle Sensitivity Analysis Delay Results ............................................ 29

Table 5.7: Pedestrian Sensitivity Analysis Delay Results ..................................................... 30

Table 5.8: Exclusive Bicycle Phase (Phases 2 and 6) Vehicle Delay Results ........................... 31

Table 5.9: Exclusive Bicycle Phase (Phases 2 and 6) Bicycle Delay Results ........................... 32

Table 5.10: Exclusive Bicycle Phase (Phases 2 and 6) Pedestrian Delay Results....................... 33 


\section{LIST OF FIGURES}

Figure 2.1: Right-hook crash types (Hurwitz, et al. 215) ………............................................. 4

Figure 2.2: Leading Bicycle Interval Sequence (MassDOT 2016)................................................ 5

Figure 2.3: Split Leading Bicycle Interval Sequence (MassDOT 2016) ........................................ 5

Figure 2.4: Exclusive Bicycle Phase Sequence (MassDOT 2016) …………………...................... 6

Figure 3.1: Study location in Portland, OR ......................................................................... 7

Figure 3.2: Real world intersection geometry and traffic volumes ........................................... 8

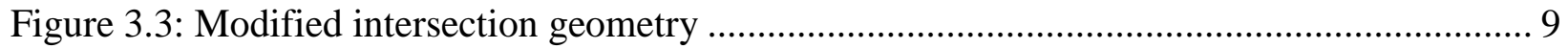

Figure 3.4: Intersection phase diagrams ................................................................................. 11

Figure 3.5: Dynamic regulatory signage (U.S. Department of Transportation: Federal Highway

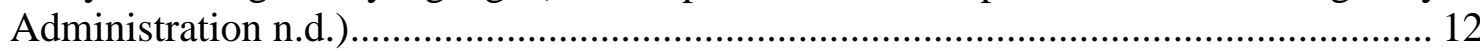

Figure 3.6: Delay gate setup ……………………………………………………………. 13

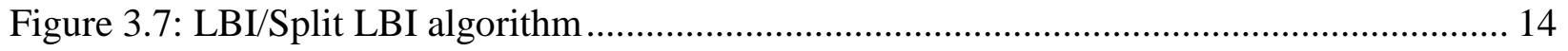

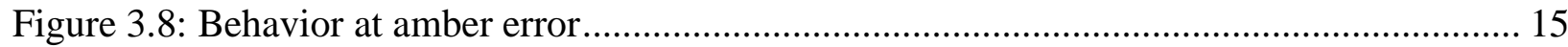

Figure 3.9: EBP phase diagram of SE $122^{\text {nd }}$ and Division ....................................................... 17

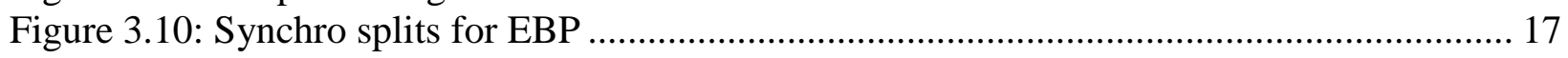

Figure 5.1: LBI vehicle thru movement delay results............................................................. 23

Figure 5.2: LBI vehicle right turn movement delay results .................................................... 23

Figure 5.3: Split LBI Vehicle Thru Movement Delay Results .................................................... 25

Figure 5.4: Split LBI Vehicle Right Turn Movement Delay Results ........................................... 25

Figure 5.5: Split LBI Bicycle Thru Movement Delay Results ………………………................. 27

Figure 5.6: Split LBI Bicycle Right Turn Movement Delay Results ........................................... 27 


\section{INTRODUCTION}

Cycling as a mode of transportation has increased in the US in recent years, from just $0.6 \%$ of all trips in 1977, to 1.0\% in 2009 (Pucher, Buehler and Seinen 2011). Many metropolitan areas have expanded their cycling facilities, and implemented bicycle share programs. Bicycling sports such as mountain biking have become incredibly popular, as have long-distance rides, and cycling-themed events. Indeed some have described the increase of cycling as a "renaissance" (Pucher, Buehler and Seinen 2011).

With higher rates of cycling, the safety risks associated with it have become more of a central concern; particularly collisions with automobiles (Dill and McNeil 2013). Bicycle vehicle collisions can be categorized into several typologies, one of which is the "right-hook" crash (Hurwitz, et al. 2015). This type of crash (see Figure 2.1) occurs when a right turning vehicle either turns in front of a through-moving bicyclist, or turns into a through-moving bicyclist. These types of crashes occur more frequently at urban intersections, including those that have traffic signals (Wachtel and Lewiston 1995).

From a traffic operations perspective (signal timing), there are options available to mitigate righthook crashes at signalized intersections. These include:

- Dynamic Right Turn on Red regulatory signage to separate right turning vehicles and bicycles.

- Leading Bicycle Intervals (LBI), where a bicyclist is given a slight head start on the vehicle traffic.

- A variation on the LBI called the Split LBI, where the bicyclist is given a head start and the non-conflicting vehicle movement(s) may also proceed.

- Exclusive Bicycle Phase (EBP), where all traffic is stopped and the bicycles are given exclusive use of the intersection.

Each of the right-hook mitigation treatments listed above have been developed primarily to increase the safety of signalized intersection for bicyclists. Limited research has been undertaken to understand the safety impacts of these treatments, but little has been done exploring how these treatments affect the performance metrics at the intersection. The delay (defined as a measurement of how much additional time is added to a road users' journey through the intersection, compared to free-flow conditions) experienced by users of the different modes is not well understood.

This study aims to understand the implications of these signal control treatments on intersection efficiency by quantifying their performance. Microsimulation will be used to model a test intersection, the results of which will be analyzed and discussed. Each of the treatments listed above (LBI, Split LBI, and EBP) will be examined and compared to a base coordinated case and impacts on all users at an intersection (passenger cars, trucks, bicycles, and pedestrians) will be analyzed.

The paper will be organized as follows: A literature review will discuss efficiency related metrics as they pertain to bicycle specific treatments at signalized intersections. This will be followed by 
a section describing the development of the simulation model. Following that the specific metrics used in the models will be discussed. An analysis of the model results will follow. Finally, a conclusion will summarize the findings. References will be located at the end. 


\section{BACKGROUND}

Increased awareness of global climate change (Stokes, Wike and Carle 2015), has led to an increasing interest in bicycling and walking in the US. Cycling as a percent of all trips has risen from just $0.6 \%$ in 1977, to $0.7 \%$ in 1990, to $1.0 \%$ in 2009 (Pucher, Buehler and Seinen 2011). Correspondingly bicycle involved crashes are also increasing in frequency. From 2003 to 2012 the number of bicyclist fatalities increased nationally from 629 to 743 , which accounts for $\pm 2 \%$ of all traffic fatalities (USDOT: NHTSA 2012). In Oregon between 2009 and 2013 there were 42 bicyclist fatalities, and in 2013 alone there were 922 bicyclist injuries (ODOT 2014). Further examination of the national data reveals that $69 \%$ of these fatal crashes happened in urban areas, and that 30\% happened at intersections (USDOT: NHTSA 2012).

Some crash types are specific to bicyclists, including the "right-hook" type crash, which is when a right turning vehicle fails to yield the right of way to a bicyclist at an intersection. These types of crashes can occur at any type of intersection (signalized or stop controlled) but can be broadly grouped into two categories (Hurwitz, et al. 2015):

1. At the start of the movement: A bicyclist and a motor vehicle are stopped together at the intersection. The driver fails to observe the bicyclist, who may be in the drivers "blind spot". When the movement starts (the signal indication turns green, or the two procced from a STOP sign) the bicyclist proceeds into the intersection, as does the right turning vehicle, thus creating the conflict. See Figure 2.1 (a) and (b).

2. After the start of the movement: One of the users, either the bicyclist or the driver, overtakes the other slower moving one. Either: the bicyclist overtakes the driver, and the driver fails to see them, and then makes a right turn into the bicyclist (Figure 2.1 (c)); or the driver overtakes the bicyclist and then turns in front of the bicyclist (Figure $2.1(\mathrm{~d})$ ).

Any given crash may have a number of contributing factors. For bicycle crashes these include, but are not limited to: driver failure to notice the bicyclist (Summala, Räsänen and Sievänen 1996), and incorrect assumptions by both drivers and bicyclists as to yielding behavior of the other (Räsänen and Summala 1998). Researchers in Oregon used a driving simulator to observe bicycle related crashes and confirmed some of these factors (Hurwitz, et al. 2015).

Design treatments are available to mitigate right-hook conflicts. These include bike boxes (aka advanced stop lines), which are used in several cities and have been shown to reduce potential conflicts (Dill, Monsere and McNeil 2010). Another mitigating treatment is the use of dynamic regulatory right turn signage, which was implemented in Portland, OR (among other places) and studied; results showed a reduction in the likelihood of right-hook crashes (Paulsen, et al. 2016).

Treatments at traffic signals, such as Leading Bicycle Intervals (LBI), Exclusive Bicycle intervals (EBP), and a newer treatment called the Split LBI, are all used today. These treatments are designed to reduce conflicts with bicyclists and turning vehicles. Outside of the realm of safety improvements, there is little research on the efficiency impacts of these treatments. This study will attempt to fill that gap by examining the performance metrics of these treatments. 


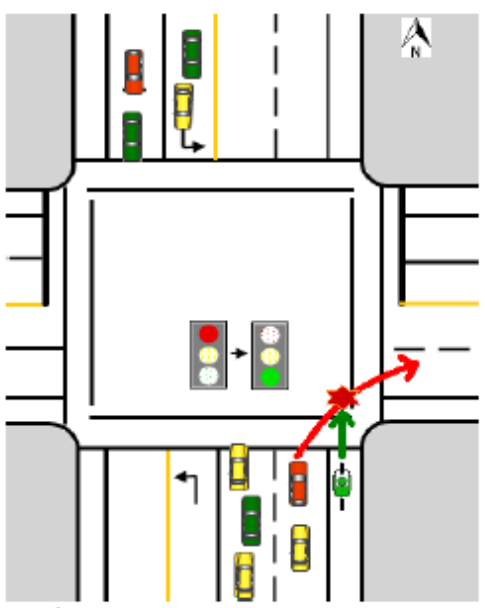

(a) Right-hook at start-up green

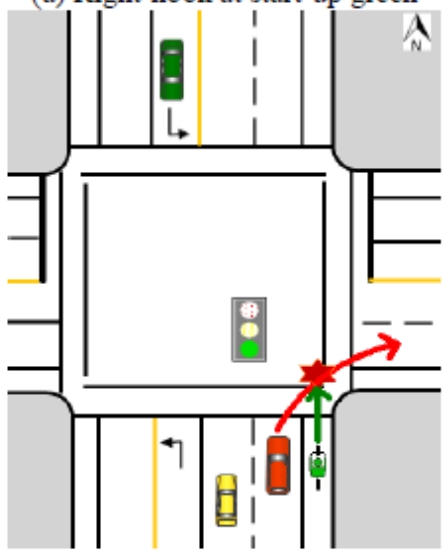

(c) Cyclist passing slow-moving car

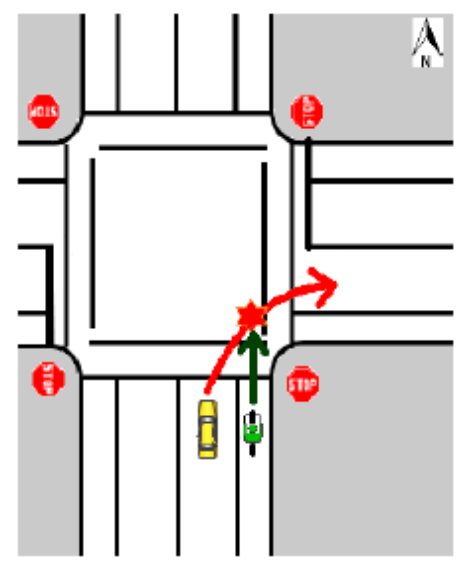

(b) Right-hook at STOP sign

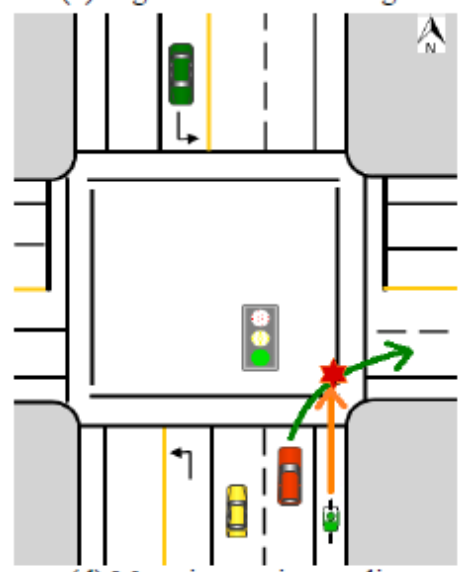

(d) Motorist passing cyclist

Figure 2.1: Right-hook crash types (Hurwitz, et al. 215)

In order to understand the treatments examined in this study each will be explained in detail. Specifics on the implementation of these treatments within the simulation program will be covered in section 3.6.

A Leading Bicycle Interval (LBI) is a scenario where bicyclists are given a head start (usually around $5 \mathrm{sec}$ ) at a signalized intersection in order to mitigate the conflicts associated with righthook type crashes. The greatest advantage of this treatment is that the bicyclists are able to establish themselves in the intersection (and in the driver's visual field), thereby reducing the probability of a collision. In order to achieve this head-start, the bicyclist is given a green indication before the vehicles in the corresponding approach. This means that all vehicles in the same signal phase are stopped, including the non-conflicting thru movements within that phase. Figure 2.2 shows an example of the LBI. 

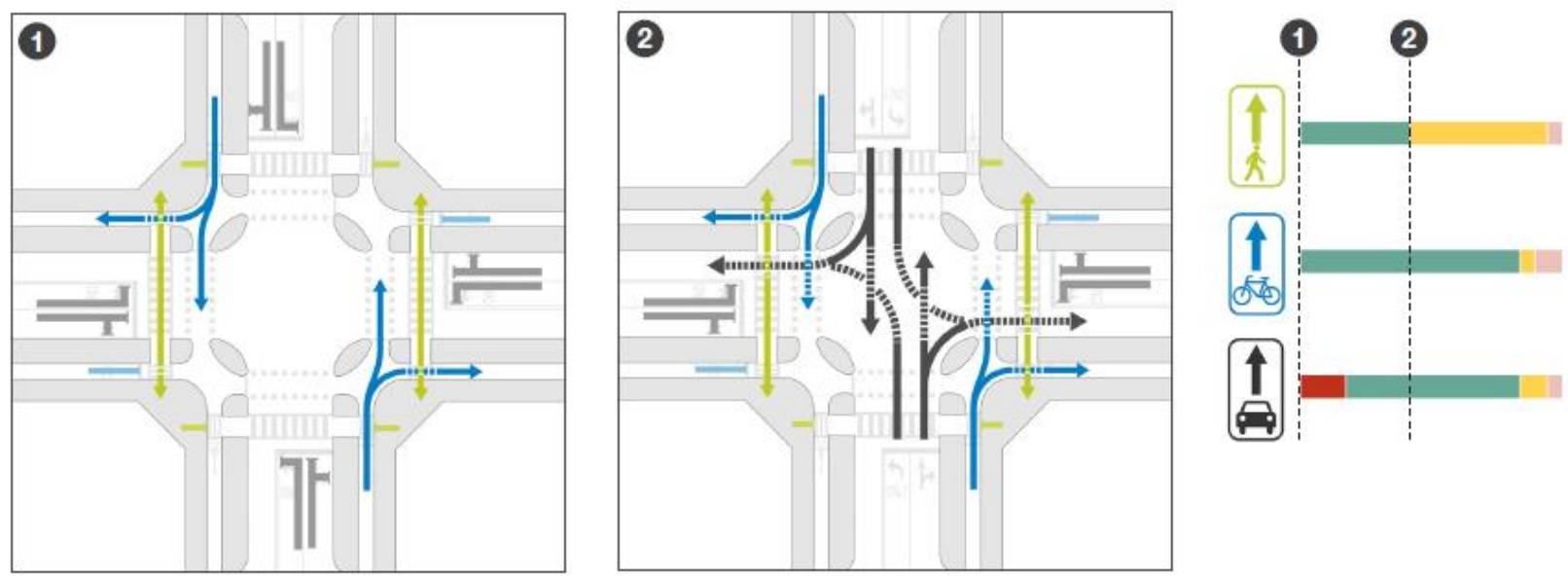

Figure 2.2: Leading Bicycle Interval Sequence (MassDOT 2016)

A variation on the LBI is the Split LBI, which is the same basic scheme but instead of stopping all the vehicles, only the conflicting right turn movements are stopped. This has the same advantages to the LBI, with the addition of allowing the thru movements to proceed without the delay from the treatment. An example can be seen in Figure 2.3.
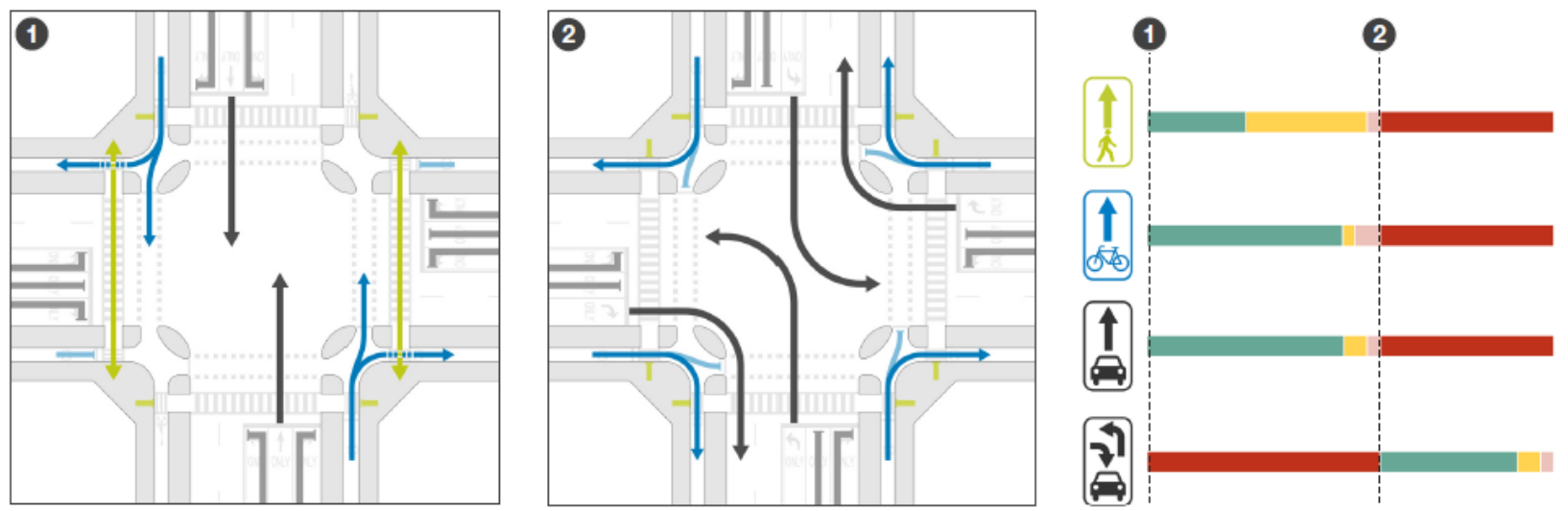

Figure 2.3: Split Leading Bicycle Interval Sequence (MassDOT 2016)

It is important to note that both the LBI and Split LBI require that motor vehicles comply with right turn on red restrictions. Unfortunately motor vehicle operators have demonstrated a low level of compliance for obeying right turn on red restrictions (Preusser, et al. 1981). Advances in regulatory signage have helped reduce these conflicts (Paulsen, et al. 2016), but the needs for the LBI and Split LBI treatments remain.

The final treatment examined is an Exclusive Bicycle Phase (EBP), which is a scheme where all traffic is stopped and the bicycles are allowed unrestricted access to the intersection (similar to an Exclusive Pedestrian Phase, also called a Barnes Dance or Pedestrian Scramble). Figure 2.4 shows an example. The EBP is often used when safety concerns dictate the need for complete separation of bicycles and vehicles. 

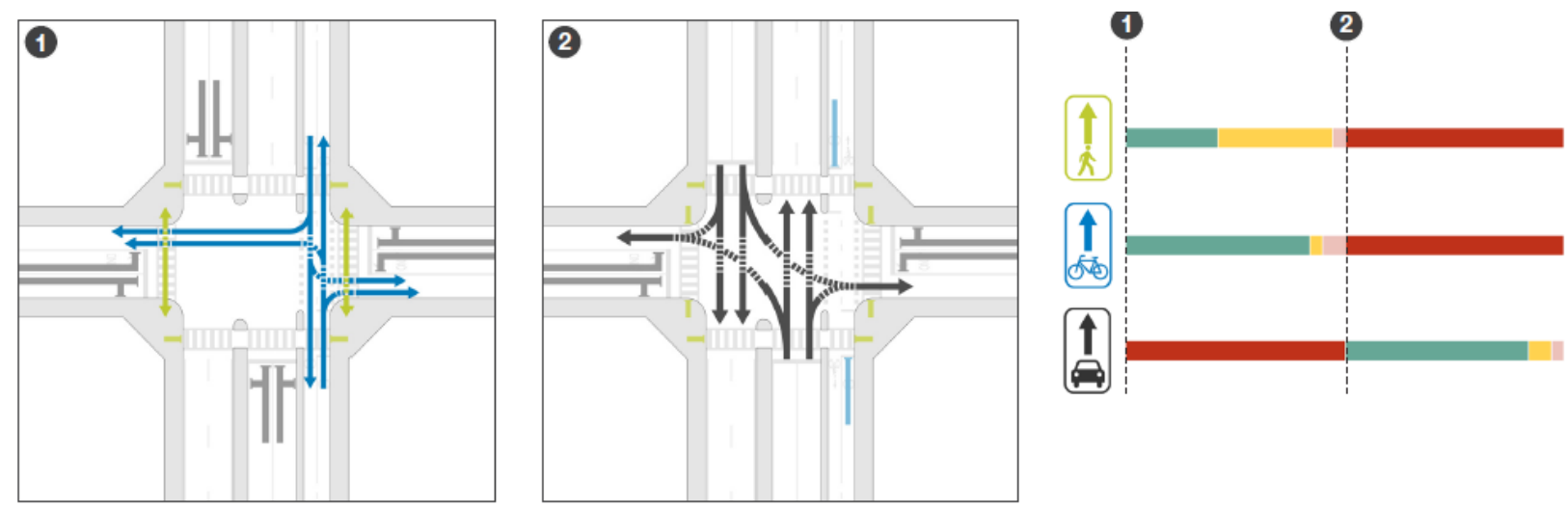

Figure 2.4: Exclusive Bicycle Phase Sequence (MassDOT 2016) 


\section{DEVELOPMENT OF SIMULATION MODEL}

In order asses the impacts of treatments (Leading Bicycle Interval (LBI), Split LBI, and the Exclusive Bicycle Phase (EBP)) on all users at a signalized intersection, microsimulation was utilized. PTV's Vissim was chosen because, of its flexibility with modeling bicyclists and pedestrians, in addition to passenger vehicle and Heavy Goods Vehicles (HGV), and for its ability to perform Software-In-The-Loop (SITL) simulation. The Econolite ASC/3 was chosen for its Programmable Logic Controller.

\subsection{Study Location}

The study location was chosen with input from project partners, especially the Portland Bureau of Transportation. The study location included three intersections along the major East-West arterial of SE Division Street. Three intersections along the corridor, 119th Ave., 122nd Ave., and 130th Ave. were modeled. Figure 3.1 shows the study location within the broader Portland area.

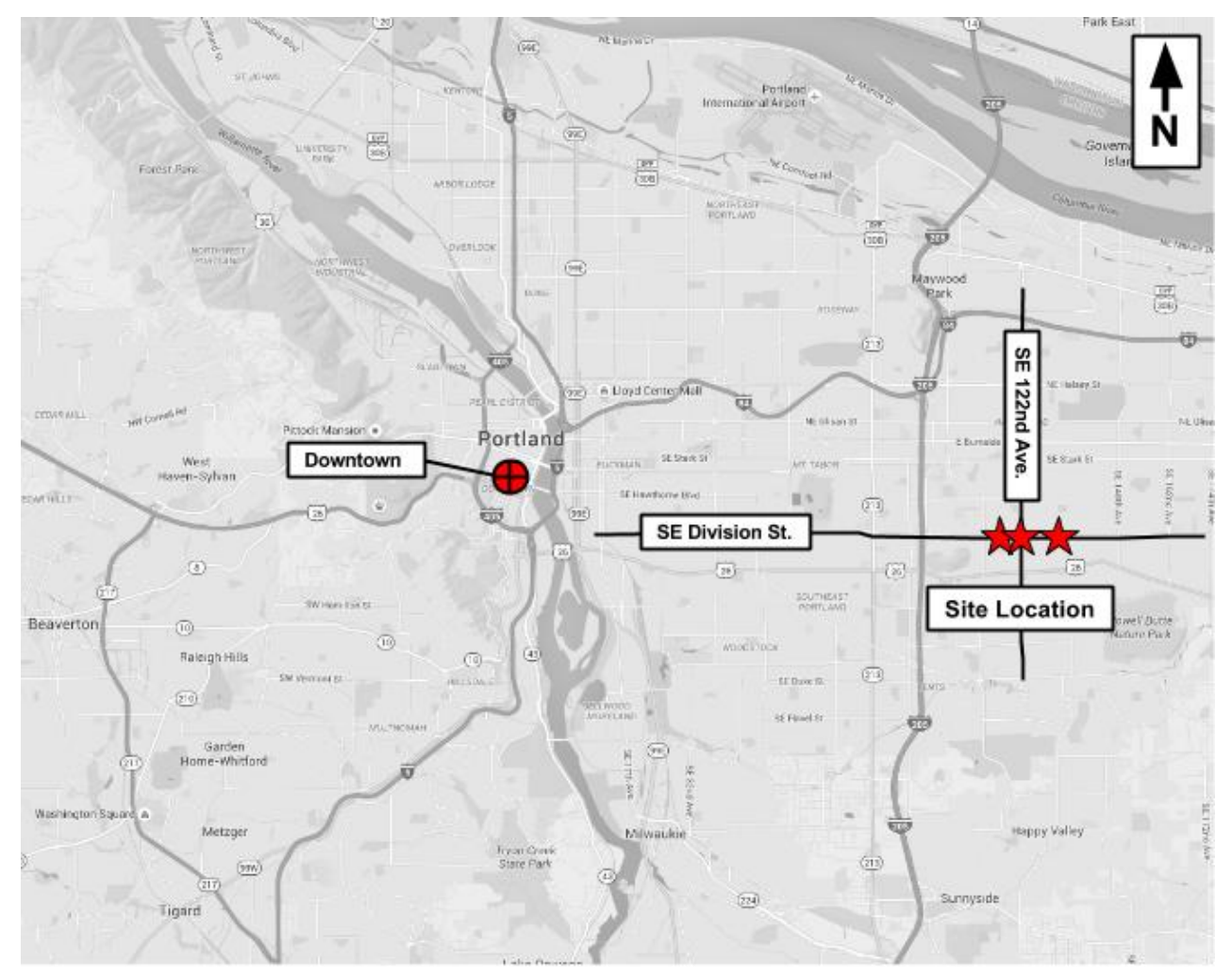

Figure 3.1: Study location in Portland, OR

These three intersections were chosen for their geometric characteristics, the ability to perform actual field implementation (which is part of a larger study, but fall outside the scope of this paper), and for the intersections location within a "High Crash Corridors" (Portland Bureau of Transportation 2014). Figure 3.2 shows the study corridor and the intersections as they currently 
exist in the real-world. This study focused on $122^{\text {nd }}$ and Division exclusively, but retained the broader network in order to increase the realism of the simulation.

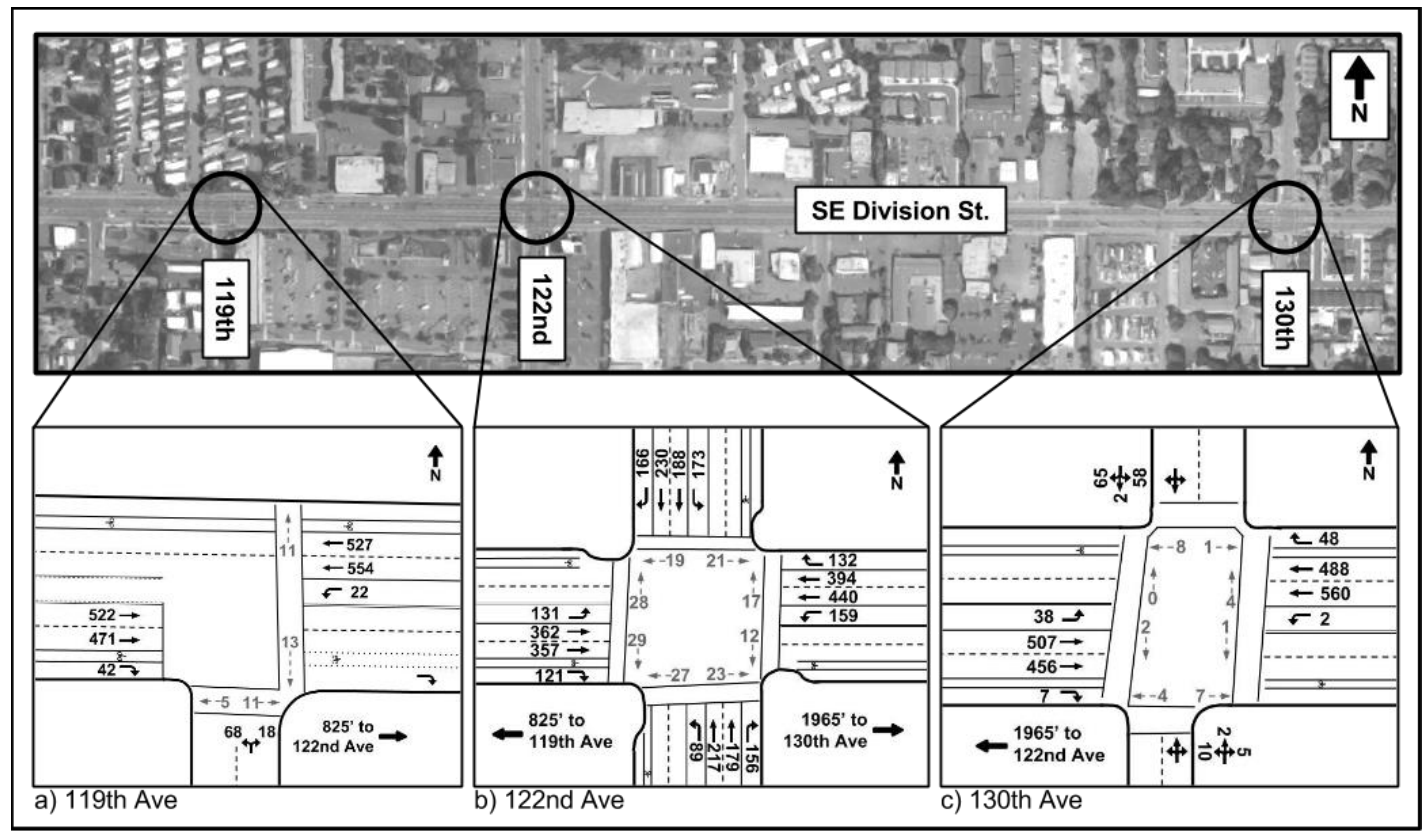

Figure 3.2: Real world intersection geometry and traffic volumes

\subsection{Geometry}

The geometric information used to build the model was obtained from several sources. Street and intersection locations, as well as placement of lanes and crosswalks, was developed using the background mapping (Bing Maps) option that is part of the Vissim software. Lane widths were found using the measuring tool in Google Maps, and were between 10' - 12' for all vehicle lanes, 5' for bicycle lanes, 10' for crosswalk widths, which were coded as two 5' parallel lanes each running in the opposite direction (north and south for example).

In order to model the conflicts between right-turning vehicles and bicycles, the geometry of SE Division St. and 122nd Ave. was modified from its actual state. First the eastbound approach was changed from an alignment where the bicycle lane was in-between the thru lane and the right turn lane, to one where the bicycle lane was to the right of the right turn lane. This was done to simulate the Split LBI treatments. The westbound approach was also changed from a dedicated right turn lane (which was a shared lane with bicycles and vehicles), to a version where the dedicated right turn lane was eliminated. In its place the bicycle lane was extended to the stop bar, and the right-most thru lane was converted to a shared thru/right-turn lane for vehicles. Figure 3.3 shows these modifications. 


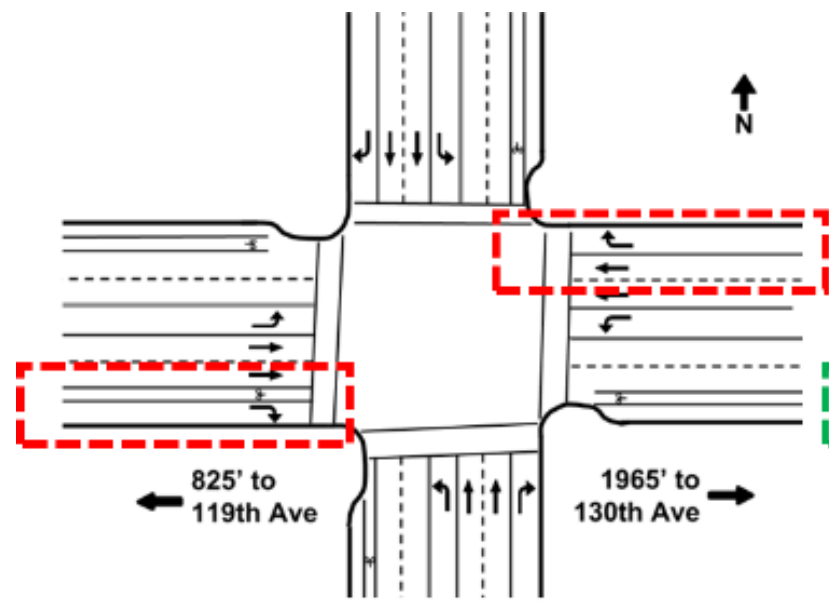

A) Real-world Geometry

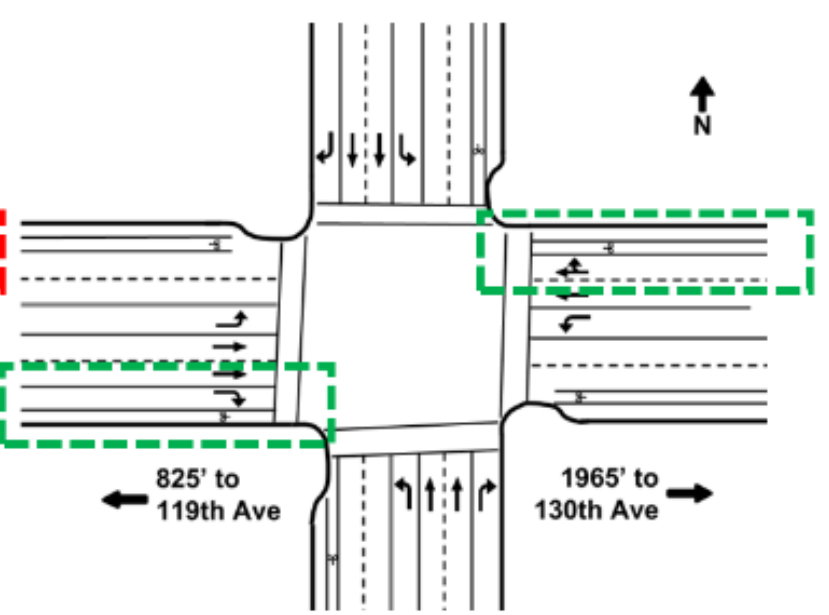

B) Altered Geometry

Figure 3.3: Modified intersection geometry

\subsection{Vehicle Volumes}

In order to simulate the treatments of the coordinated base case, LBI, Split LBI, and EBP, Vissim required the user to input traffic volumes, vehicle types, and speeds. Traffic volumes, and vehicle compositions were obtained from Quality Counts, a traffic data collection firm. The data collection was performed on Tuesday September 22nd 215 and included: 24-hr tube counts (including vehicle classification and volumes); and turning movement counts form 11:00 a.m. to 1:00 p.m. (which included bicycle and pedestrian counts) at all three intersections.

Results showed a total average daily vehicle count of $\pm 18000 \mathrm{veh} /$ day. Figure 3.2 shows the volumes during the study time interval (with the volumes remaining the same despite the geometric changes). HGVs varied between $3 \%-10 \%$ for the corridor. Pedestrian volumes were 184 at the peak $15 \mathrm{~min}$ period from 12:45 p.m. to 1:00 p.m. at 122nd, while bicycle counts totaled three for the same period.

The Quality Counts data had two aspects that required modification for modeling: many bicycle movements were unused during the count (listed as zeros in the tally), and the pedestrian data was recorded only for the crosswalk used, but not the pedestrian's direction of travel.

The bicycle volumes in the Quality Counts data were so low that many of the movements had zero bicyclists, while the others had as little as one bicyclist. To remedy this a sensitivity analysis was devised (see section 4.2 below)

Pedestrians used in Vissim require detailed information on their movements for proper coding, this includes not only the crosswalk used but also the direction of crossing. The pedestrian movement data collected by Quality Counts only coded the number of pedestrians using each crosswalk (for example the north side had 37 crossing, but no directional data), meaning that counts in each direction were not available. To resolve this discrepancy video data from a prior traffic count at the intersection that was done in June 2015 was used. In the previous traffic 
count, video data was recorded for a $24-\mathrm{hr}$ period and the pedestrians crossing movements were observed and tallied. These results included the direction of the pedestrian movement, for example crossing from east-to-west or from west-to-east. Those directional ratios from the previous pedestrian movement study were then applied to the Quality Counts pedestrian count data, thereby giving a reasonable estimate for the number of pedestrians crossing in a given direction.

\subsection{Speeds}

Vissim uses mathematical distributions for speed as defined by the user. Table 3.1 shows the posted speeds for the streets in the model, the distribution used for each of those streets, and the speed distribution for each mode type. It was also assumed that right turning vehicles make the turn at $9 \mathrm{mph}$, while left turning vehicle used $15 \mathrm{mph}$.

Table 3.1: Speeds in model

\begin{tabular}{ccccc}
\hline & SE Division & SE 119th & SE 122nd & SE 130th \\
\hline Posted Speed (mph) & 35 & 25 & 35 & 25 \\
Vissim Speed Distribution Range (mph) & $33-37$ & $33-37$ & $23-27$ & $23-27$ \\
& Car & HGV & Bicycle & Pedestrian \\
\cline { 2 - 5 } Speed Distribution by Mode (mph) & By Speed Limit & By Speed Limit & $8-12$ & $3-5$ \\
\hline
\end{tabular}

Transit operations, though present in the field, was excluded from the models in order to simplify the total number of variables that would influence the study. In addition, no vehicle occupancy data was obtained and therefore vehicle occupancy was assumed to be 1 person per vehicle.

\subsection{Driver Behavior}

Vissim utilizes mathematical functions to model the behavior of users (pedestrian, bicycle, vehicle) in the simulation (PTV Group 2014). These include behavioral factors such as lane changing, lateral motions, and actions related to traffic signals, including the way a vehicle behaves at an amber indication (called "Behavior at Amber"). Default settings in Vissim were used for all behaviors with the exception of Behavior at Amber (see section 3.6.1.1.2 below).

\subsection{Signal Control}

All three of the intersections in the study were signalized. Timing plans, detector plans, and other pertinent information were provided by the Portland Bureau of Transportation (PBOT). For each of the treatments Synchro Traffic Modeling software was used to develop the coordination specifics including the timing splits and offsets. An additional alteration was the use of the 
Econolite ASC/3 controller in place of the Type 270's and 170's with Voyage and Wapiti software that were present at the intersections. This was done to take advantage of a native Programmable Logic Controller (PLC) present in the ASC/3, which was used for the implementation of the bicycle specific treatments.

The intersection of SE119 ${ }^{\text {th }}$ was a T-intersection and used phases $1,2,6$, and 8. 119th had no North side approach (Figure $3.4 \mathrm{~A}$ ), as such it also lacked the eastbound left turn lane (Phase 5), the westbound right turn lane (Phase 6 right turn), the northbound thru lane (Phase 8 thru), and lacked two of the pedestrian crossings. Figure 3.4 B shows the phase diagram for the intersection of SE Division and $122^{\text {nd }}$, which was as standard 8-phase intersection where phases 2 (eastbound) and 6 (westbound) were the major phases, while phases 4 (southbound) and 8 (northbound) were the minor phases. $130^{\text {th }}$ (Figure $3.4 \mathrm{C}$ ), used a 6-phase configuration, lacking the dedicated left turn phases on the minor approaches and the right turn lanes on the major compared to $122^{\text {nd }}$.

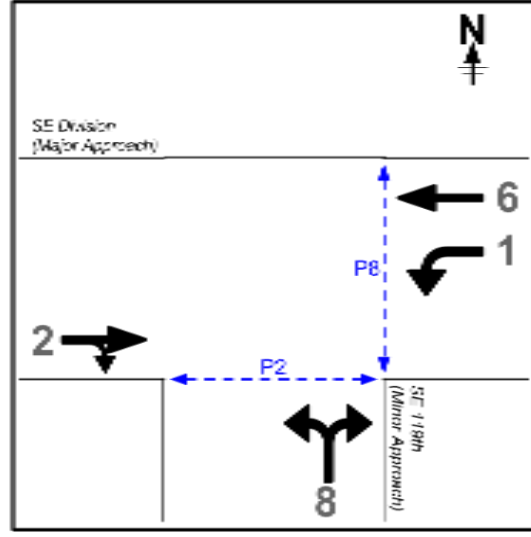

A) SE 119th

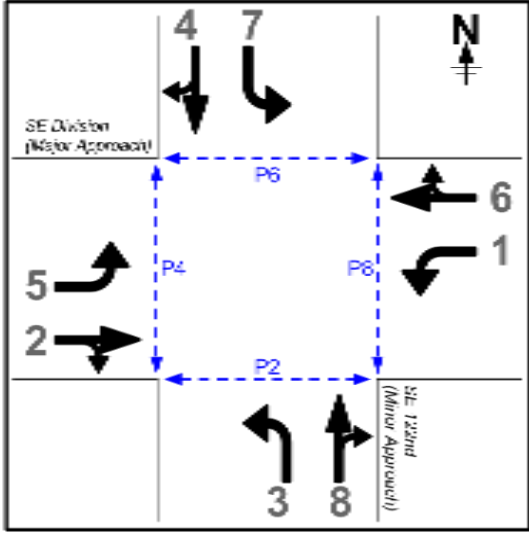

B) SE 122nd

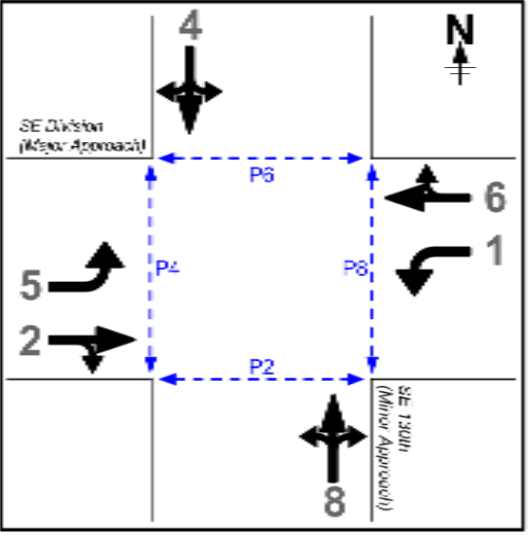

C) SE 130th

Figure 3.4: Intersection phase diagrams

All right turn movements at the intersection allowed Right Turn on Red (RTOR), except during the time the LBI and Split LBI treatments were active (see 3.6.1.1). Another notable change to the signalization along the study corridor was the development of a coordination scheme. The study corridor is not normally run in coordination, where all three intersections, $119^{\text {th }}, 122^{\text {nd }}$, and $130^{\text {th }}$, would be coordinated with each other. In practice the intersection of $119^{\text {th }}$ and Division is coordinated with the intersection of $112^{\text {th }}$ (which was not included in the study corridor), the intersection of $122^{\text {nd }}$ is set to Free, and the intersection of $130^{\text {th }}$ is coordinated with $136^{\text {th }}$ (which was not included in the study corridor).

The change was made to compare the impacts of the treatments against the coordinated base case, which is why all three intersections were placed in coordination with each other.

For the coordination scheme a cycle length of $110 \mathrm{sec}$ was chosen. The cycle length varies at $122^{\text {nd }}$ and Division throughout the day, allowing for responsive operation during peak hours. A $110 \mathrm{sec}$ cycle length was used to correlate with the common pedestrian peak hour of $\pm 12: 00$ p.m., which at that time of day runs at $110 \mathrm{sec}$. 


\subsubsection{Leading Bicycle Interval and Split Leading Bicycle Interval}

The LBI and Split LBI required the use of a special setup in Vissim. Several elements were added to the model or changed to accommodate the treatments, these include: an additional set of signal heads to control traffic during the LBI (termed "Delay Gates"), the use of a Programmable Logic Controller (PLC), an alteration to the way vehicles interact with an amber indication, and the alteration of the bicycle volumes present at the intersection of $122^{\text {nd }}$. Each of these will be explored in depth below, beginning with the Delay Gates.

\subsubsection{Delay Gates}

In practice LBI and Split LBI treatments require a method to control right-turning traffic during the treatment itself. Often this is achieved by using signage, such as "No Turn on Red" signs, or by using dynamic regulatory signage (Figure 3.5 ). Since Vissim does not offer a conditional RTOR setting, a separate system was devised to enforce the LBI and Split LBI treatments. This system involved the creation of a second set of signal heads that in effect mimicked a Dynamic Regulatory Right Turn Sign.

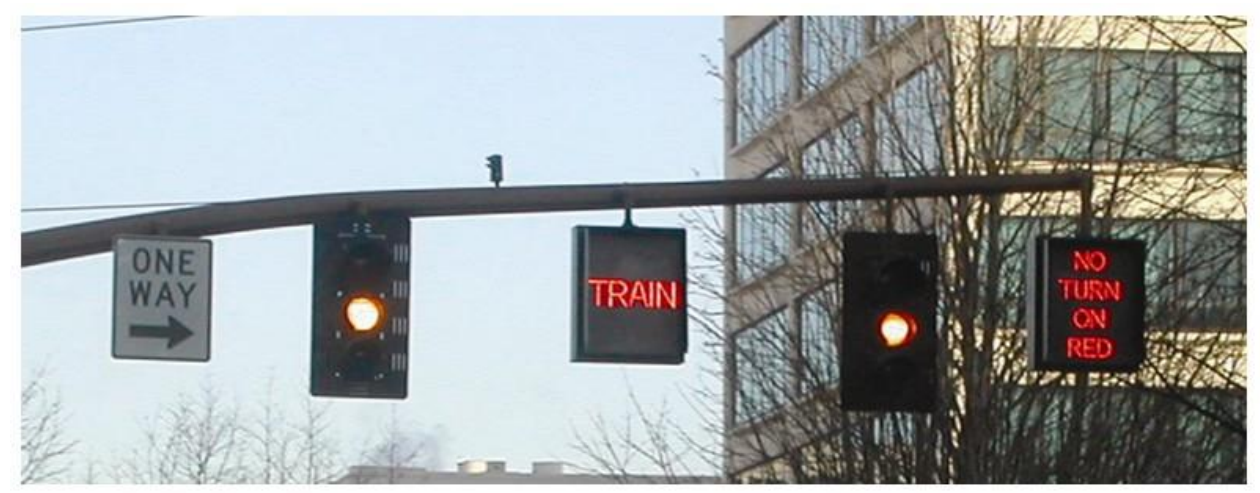

Figure 3.5: Dynamic regulatory signage (U.S. Department of Transportation: Federal Highway Administration n.d.)

These new signal heads were termed "Delay Gates" and their setup in Vissim can be seen in Figure 3.6 below. The system used the Programmable Logic Controller (PLC) present in the $\mathrm{ASC} / 3$ to change the indication of the Delay Gate from a rest-state of green, to red during the 5 sec of LBI or Split LBI. 


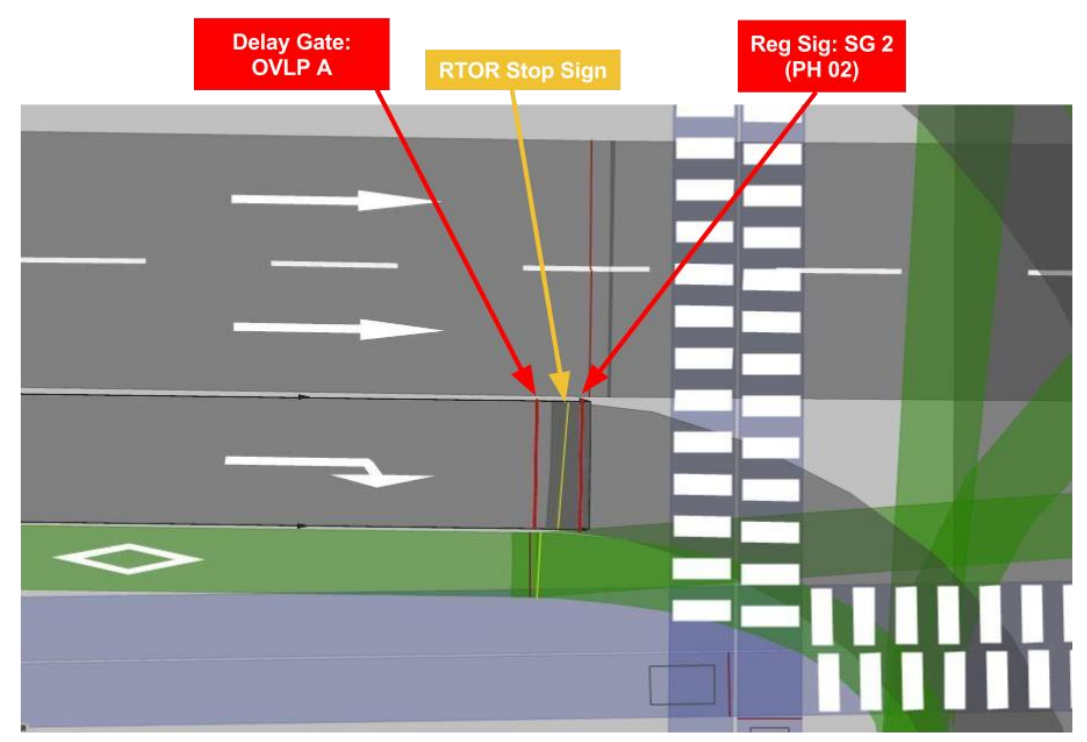

Figure 3.6: Delay gate setup

These Delay Gates effectively simulated dynamic regulatory right turn signage. Both eastbound $\&$ westbound used the same basic scheme, but applied to different lanes as appropriate to the LBI or Split LBI. The Delay Gate was placed in the eastbound right turn lane for the Split LBI, and the westbound thru and shared thru/right turn lanes for the LBI. They were located slightly upstream of the main vehicle signal head and the RTOR stop sign. They operated by turning red only during the LBI, thereby restricting the vehicle movements from proceeding in their regular path, especially from making right turns (i.e. potential right-hooks). The gates were assigned to Overlaps A (phase 2) and C (phase 6).

The LBI operated in the following sequence (See Figure 3.8 below): First the regular signal heads turn from green, to amber, to red at the end of their phase. Just before the start of the vehicle phase the Delay Gates turns from green, to amber, to red (thus beginning the LBI/Split LBI). Quickly thereafter the regular signal heads turn green (starting the vehicle phase), however the vehicles are restricted by red Delay Gate. After $5 \mathrm{sec}$, the Delay Gates turns green, and the vehicles are no longer delayed. This marks the completion of the LBI/Split LBI cycle.

\subsection{LBI and Split LBI Algorithm}

An algorithm was developed that worked in tandem with the Delay Gates (i.e. additional signal heads) to create the LBI treatments. The algorithm took advantage of Overlaps and the PLC capabilities within the ASC/3 controller.

Overlaps A and C were used to control the LBI. Both were set such that all phases were turned on; Overlap A included phases 1, 2, 3, 4, 5, 6, 7, and 8. Overlap C was the same. This allowed the signal-state to remain green at all times, and then be "Terminated" in logic step \#3 (See Figure 3.7), causing the indication to change from green to red for the duration of the LBI or Split LBI. 

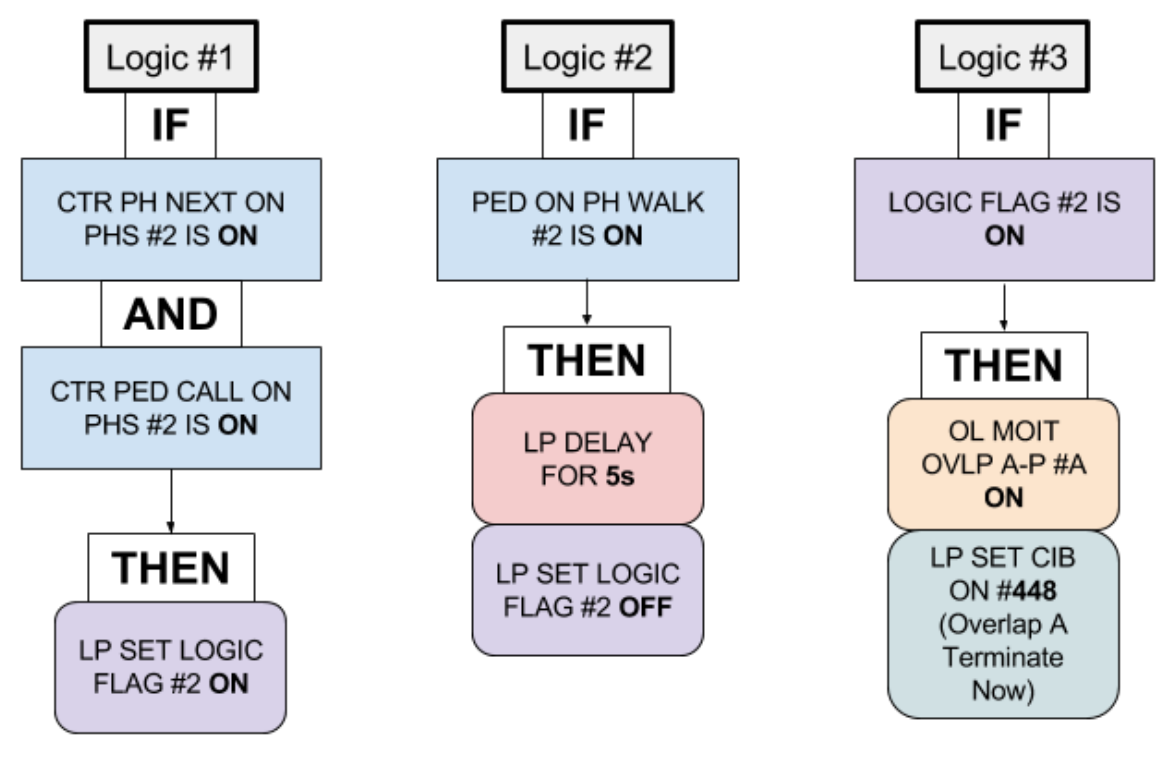

Figure 3.7: LBI/Split LBI algorithm

The logic statements in Figure 3.7 can be explained further as follows: When the WALK signal illuminates, the overlap turns red (the always-green Delay Gate turning to red is the overlap being "Omitted"), and then delays that for $5 \mathrm{sec}$. After the $5 \mathrm{sec}$ the Delay Gate turns green again, and the cycle repeats itself.

The PLC uses the pedestrian timing in logic \#1 and \#2 because the LBI and Split LBI phases are set to Pedestrian Recall, meaning that the pedestrian movements will be served every cycle. This provides an easy marker to tie the logic functions to.

\subsection{Behavior at Amber}

Vissim allows the user to pick between two options for the Behavior at Amber: "Continuous Check" and "One Decision" (PTV Group 2014). Continuous Check allows the vehicle in the model to continuously check (checks every 2 seconds) the status of the signal state and then decide whether to go or not. One Decision uses a probabilistic function to decide whether or not to stop at the amber, and when a decision is made it is not re-examined. The main difference between the two is that Continuous Check gives more opportunity to pass through the signal during the amber illumination.

A quirk in the operation of the LBI and Split LBI treatments (see section 3.6.1.1) compelled an alteration of the Behavior at amber. During testing it was observed that vehicles could "sneak" through the LBI treatments. Figure 3.8 shows the following sequence: 

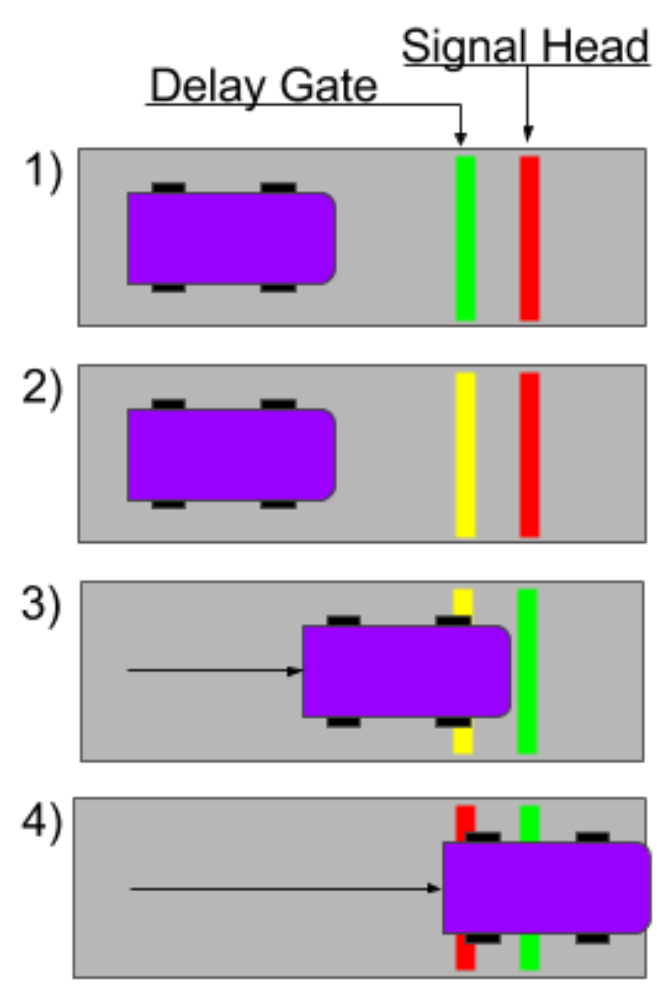

Figure 3.8: Behavior at amber error

1. The stopped vehicle observes the red indication and does not advance.

2. Delay Gate turns amber, vehicle makes first Decision at Amber. Vehicle does not proceed because the signal head is still red.

3. Just over $2 \mathrm{sec}$ later the Delay Gate is still amber and the Signal Head turns green, since 2 sec has elapsed the Decision at Amber rechecks, and finding a green indication on the Signal Head rather than a red indication as before, proceeds.

4. A split second later the Delay Gate turns red, but the Decision at Amber has been made and the vehicle is already advancing, negating any benefit from the LBI.

Changing the Decision at Amber from Continuous Check to One Decision remedied the problem. In the scenario above the vehicle completes step one by making the One Decision, but then remains stopped and does not advance during the potential operation flaw in step 3 .

Since the behavior at amber was changed from its default Continuous Check to One Decision, the change was examined to see what occurred as a result. Results indicated that delay increased slightly, which is expected. The amber time at $122 \mathrm{nd}$ is $3.9 \mathrm{sec}$ for the through phases. The Continuous Check checks every two seconds (thereby allowing at least 2 checks for each amber), while the One Decision option only checks the signal state once. During One Decision the vehicle has fewer opportunities to evaluate the signal state, and in turn will be more likely to stop at the amber, which in turn increasing delay.

The changes associated with altering the behavior to amber can be seen in Table 3.2, which shows the percent difference in user delay (\% Diff) when comparing the Continuous Check and 
One Decision options, where "LBI: RT" (westbound right turn; the highest change at 4.3\%) represented a $1.4 \mathrm{sec}$ increase in total user delay. Other values differ by tenths of a second. This suggests that changing the Behavior at amber had little overall impact on user delay. It is therefore assumed that the changing of the Behavior of Amber was an appropriate alteration for the circumstances.

Table 3.2: Decision at amber change analysis

\begin{tabular}{ccccc}
\hline Movement & $\begin{array}{c}\text { Continuous Check } \\
(\text { sec })\end{array}$ & $\begin{array}{c}\text { One Decision } \\
(\text { sec })\end{array}$ & \% Difference & $\begin{array}{c}\text { Delay Difference } \\
(\text { sec })\end{array}$ \\
\hline Split LBI: TH & 16.83 & 16.93 & $0.6 \%$ & 0.1 \\
Split LBI: RT & 5.31 & 5.32 & $0.2 \%$ & 0.1 \\
EB LT & 62.57 & 63.24 & $1.1 \%$ & 0.67 \\
LBI: TH & 28.15 & 28.63 & $1.7 \%$ & 0.48 \\
LBI: RT & 24.13 & 25.17 & $4.3 \%$ & 1.4 \\
WB LT & 52.45 & 53.17 & $1.4 \%$ & 0.72 \\
NB TH & 37.32 & 37.42 & $0.3 \%$ & 0.1 \\
NB RT & 7.21 & 7.23 & $0.3 \%$ & 0.2 \\
NB LT & 55.53 & 56.1 & $0.9 \%$ & 0.48 \\
SB TH & 34.55 & 34.47 & $-0.2 \%$ & -0.8 \\
SB RT & 6.17 & 6.15 & $-0.3 \%$ & -0.2 \\
SB LT & 55.09 & 55.92 & $1.5 \%$ & 0.83 \\
122 & 29.89 & 30.17 & $0.9 \%$ & 0.28 \\
\hline
\end{tabular}

\subsubsection{Exclusive Bicycle Phase}

Another treatment studied was an Exclusive Bicycle Phase (EBP), which is a scheme where all traffic is stopped except for the bicycle traffic. From an operational standpoint the EBP was implemented by adding Phase 9 (which corresponded to Bike Phase 2) and Phase 10 (corresponded to Bike Phase 6) at the end of rings I and II (See Figure 3.9). This allowed the EBP to time as a separate phase pair. 


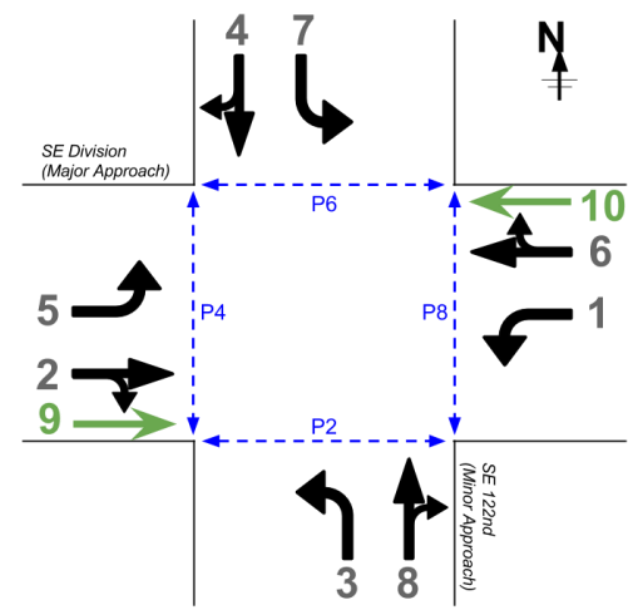

Figure 3.9: EBP phase diagram of SE $122^{\text {nd }}$ and Division

In addition, the following were programmed: $5 \mathrm{sec}$ amber (needed to clear $10 \mathrm{mph}$ bicycles from the intersection); a $1 \mathrm{sec}$ red Clearance; a $10 \mathrm{sec}$ green time (splits); and (since the bicycle approaches had detection) a 2 sec Vehicle Ext time.

In order to ensure an "apples-to-apples" comparison between the base case and the EBP the cycle length remained at $110 \mathrm{sec}$; thereby eliminating the effects of differing cycle lengths from complicating the analysis. This EBP effectively caused an approximately $15 \mathrm{sec}$ shortening of the overall cycle length; this reduction in cycle length took away green time from the other phases and gave it to the EBP. At $10 \mathrm{sec}$ of EBP green time, only three bicyclists were able to pass through the signal head.

One notable challenge that was encountered was the development of the timing scheme for EBP implementation. Synchro was used to develop the timing plans, but the software does not have the ability to model bicyclist as active users of the roadway, they are instead coded in the program as an interference for vehicles. In order to resolve this, the EBP was modeled as an Exclusive Pedestrian Phase (which is a setting that Synchro does have) with a value of 10 sec. Figure 3.10 shows the Synchro splits, with the EBP seen as the pedestrian Phase 09 at the end of the cycle. From this model offsets and splits were obtained, which were used in the EBP Vissim model.

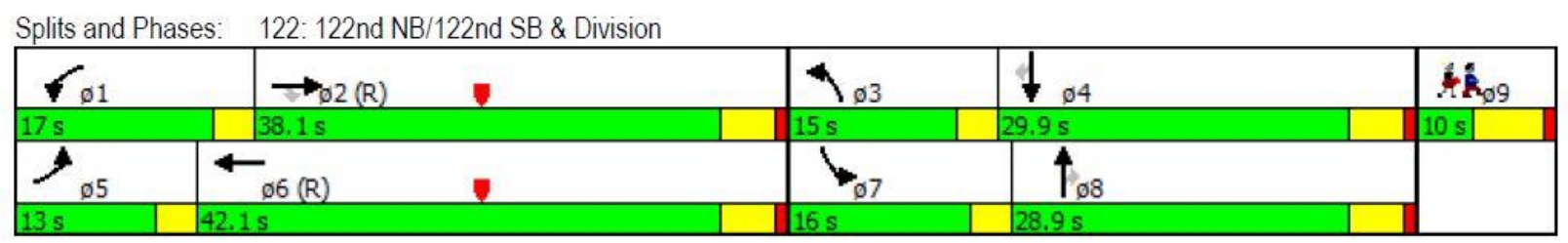

Figure 3.10: Synchro splits for EBP

The simulation of the EBP did not include the sensitivity analysis like the LBI treatments did, this was due to the single file bicycle lane setup, which severely limited the realism of the EBP. Bicyclists, because of the nature of their vehicle, do not queue up like motorized vehicles do; 
they tend to "pack in" much tighter. The model retained the same vehicle queueing behavior for bicycles, passenger cars, and HGVs.

With regards to EBP performance this means that the way Vissim models the bicyclists during the $10 \mathrm{sec}$ of EBP only allows for three to pass through the light. This is probably lower than what could be expected for a real life intersection, which would imply that the delay values seen in the results could be improved with modifications to the queuing-at-stop behavior of the bicyclists (see section 6.1 below).

The inability to move more than three bicyclists through the intersection during the EBP limited the upper bounds of the bicycle volumes, which is why the sensitivity analysis was not completed for this treatment. However, the Quality Counts volumes from September were too low to produce meaningful data results. In order to remedy this conundrum a bicycle volume of $1 \%$ of vehicle traffic was used, which was compared to the base case $1 \%$ scenario. 


\section{METHODS}

In order to test the proposed treatment types at the intersection of $122^{\text {nd }}$ and Division the Vissim model was coded for specifics such as number of runs and randomization of the vehicles. Additionally, a base case model was developed and validated using a state DOT protocol. Bicycle volumes were also altered to accommodate shortcoming in the traffic data, and the model results were tested for statistical accuracy using T-Tests. Specifics of these will be explored in depth below.

\subsection{Model Calibration}

Simulation models are often calibrated to real world data in order to make estimates on their accuracy. The Oregon Department of Transportation (ODOT) uses the GEH formula to compare the real world input volumes and model output volumes. The formula is given by:

$$
G E H=\sqrt{\frac{2(m-c)^{2}}{m+c}}
$$

where,

$\mathrm{m}=$ output traffic volume from simulation model (vph)

$\mathrm{c}=$ input traffic volume $(\mathrm{vph})$

The ODOT VISSIM protocol report provides guidance on acceptable values for GEH statistic (ODOT 2011). The criteria are listed in Table 4.1. ODOT recommends that GEH statistics should be calculated for all intersection turns and mainline links and for traffic volumes at all entry and exit locations for each model, with the criteria presented in Table 4.1 used to assess the validity of the model results.

\section{Table 4.1: ODOT GEH criteria}

\begin{tabular}{l|l}
\hline Value of Statistic & Criteria \\
\hline $\mathrm{GEH}<5.0$ & Acceptable fit \\
$5.0<=\mathrm{GEH}<=10.0$ & Caution: possible model error or bad data \\
$\mathrm{GEH}>10.0$ & Unacceptable \\
\hline
\end{tabular}

(Source: ODOT, Protocol for VISSIM Simulation, June 2011)

The model was validated using the base case volume outputs for vehicles and pedestrians (bicycles were not validated because of the bicycle volume sensitivity analysis) and the input volumes from the Quality Counts data. The GEH analysis revealed GEH $<5.0$ for all vehicle and pedestrian links, meaning all data was considered to have an "Acceptable Fit".

\subsection{Number of Runs}

Vissim allows users to define the model's parameters for simulation (number of runs, randomizer information, and total time interval of the runs), and how the model will vary those parameters. 
In order to obtain meaningful statistical information from the treatments, each model (base case, LBI, Split LBI, EBP) was run 10 times. A randomizer inherent in the program varies aspects like vehicle arrivals and volumes, and does so by user defined "seed" intervals. A random seed interval was used, starting at random seed number 56, and increasing the seed value by 1 each run. The duration of each run was 4,500 sec ( $1 \mathrm{hr}$ and $15 \mathrm{~min}$ ), but was only recorded for the last $3,600 \mathrm{sec}(1 \mathrm{hr})$. This allowed the model time to populate with traffic.

\subsection{Metrics}

Vissim allows for the gathering of a number of traffic performance metrics, including: queueing, delay, travel times, as well as logging capabilities for signal changes, detector calls, and many other features. For this study delay per user, number of users, queue length, and several other metrics were recorded. These were gathered from "nodes", which are data gathering boundaries set by the user in Vissim. The nodes were drawn by the modeler as squares that surrounded the intersection, thereby limiting data collection to just the intersection itself. Vissim sorts all metrics by the movement and vehicle type.

\subsection{Statistics}

In order to determine the validity of the results a standard paired, two-tailed, T-Test was performed using Microsoft Excel. The two sample T-Test statistically examines if the means of two populations are different. The test assumes a normal distribution and is performed when the sample size is small. The formula is listed below:

Where

$$
t=\frac{\overline{x_{1}}-\overline{x_{2}}}{\sqrt{\frac{s_{1}^{2}}{n_{1}}+\frac{s_{2}^{2}}{n_{2}}}}
$$

$\overline{x_{1}}=$ Mean 1

$\overline{x_{2}}=$ Mean 2

$s_{1}=$ Standard deviation 1

$s_{2}=$ Standard deviation 2

$n_{1}=$ Total sample size 1

$n_{2}=$ Total sample size 2

All results from the statistical analysis were incorporated into the data tables in the Results section. The results were tested for significance at the $95 \%$ confidence interval (CI) using the Microsoft Excel =t.test () function.

\subsection{Bicycle Volumes}

Bicycle volumes at the intersection were low, eight total for the study hour of 12:00 p.m. to 1:00 p.m. This caused two issues with the model: there were not enough bikes to fully test the 
treatments, and the results for bicyclists were suspect due to small sample size errors. In order to remedy this, a sensitivity analysis was performed where bicycle volumes were varied as a function of vehicle volume, from $1 \%-10 \%$, in $1 \%$ increments. This analysis was performed for the LBI, the Split LBI, and for the base case. The additional bicycles were added to the model (as opposed to removing vehicles to maintain the same overall volume of road users), increasing the total number of users. The number of bikes is shown in Table 4.2 below.

Table 4.2: Sensitivity analysis bicycle volumes

\begin{tabular}{cccc}
\hline \% Bikes & Number of bikes & In at 119th $(\boldsymbol{E B})$ & In at 130 $(\boldsymbol{W B})$ \\
\hline $1 \%$ & 36 & 18 & 18 \\
$2 \%$ & 71 & 36 & 36 \\
$3 \%$ & 17 & 53 & 53 \\
$4 \%$ & 143 & 71 & 71 \\
$5 \%$ & 178 & 89 & 89 \\
$6 \%$ & 17 & 17 \\
$7 \%$ & 214 & 125 & 125 \\
$8 \%$ & 250 & 143 & 143 \\
$9 \%$ & 285 & 160 & 160 \\
$10 \%$ & 321 & 178 & 178 \\
\hline
\end{tabular}

The sensitivity analysis posed a second challenge: what to do about bicycle movements from the Quality Counts data that had zero bicyclists? It was decided to not alter the bicycle volumes on the minor approaches, and to adopt a scheme for the major treatment approaches where $15 \%$ of bicyclists turned right, and 15\% turned left, while the remaining $70 \%$ used the thru movements. The right turning bicyclists turned from the bike lane into another bike lane. The left turning bicyclists merged across traffic, using the left vehicle turn lane to complete the movement into the destination bicycle lane.

\subsection{Coordinated Base Case}

In order to set the datum to which the treatments would be compared, a "base case" scenario was developed. This base case used the modified intersection geometry, volumes, and other parameters noted above. Once the base case was completed it was copied and the individual treatments were implemented into that copy, thereby ensuring valid comparisons. It was decided to adopt a coordinated signal strategy, and to develop the necessary signal timing using a combination of PBOT provided timing plans and Synchro traffic modeling software. Although $122^{\text {nd }}$ and Division was at one time run in coordination, it runs in free mode at present. Because of this the coordination had to be re-developed. The results taken from Synchro included splits and offsets. 


\section{ANALYSIS AND RESULTS}

The four simulation scenarios, LBI, Split LBI, EBP, and base case coordinated, were modeled in 10 run sets using Vissim. The base case scenario was then compared to the LBI, Split LBI, and EBP in order to gauge the changes due to the treatments. Due to low bicycle volume the LBI and Split LBI results contain a sensitivity analysis where the number of bicycles was increased as a function of the vehicle volume. The EBP used only the $1 \%$ volume scenario from the sensitivity analysis. The results of these will be explored below.

\subsection{Coordinated Base Case}

In order to establish the datum to which the test treatments would be compared, a base case scenario was modeled. This base case used the same modified geometry at 122nd and Division and was identical to the treatment scenarios (minus the treatments themselves). This includes using the same volumes as the treatments, including the bicycle volume sensitivity analysis. All base case results can be seen in the treatment comparisons within the following sections.

\subsection{Leading Bicycle Interval (LBI)}

In this research both LBI scenarios were examined simultaneously. The eastbound approach (phase 2) utilized the dedicated right turn lane and bike lane (which extended to the stop bar), to implement the Split LBI. The westbound approach (phase 6) lacked a dedicated right turn lane, necessitating the stopping of the entire phase during the LBI. This allowed the testing of both LBI and Split LBI treatments simultaneously. The modeling software, Vissim, parses out the results of each movement, giving a simple method for extracting the metrics related to each LBI/Split LBI treatment. Because of the low bicycle volumes present at the intersection a sensitivity analysis was performed where the bicycle volume was increased as a function of the percent of mode share. The actual number of bicycles can be seen in Table 4.2. The results of each treatment have been separated for analyses, and will be discussed individually below.

\subsubsection{Traditional LBI (Westbound)}

The westbound approach of the model (Phase 6) used the LBI treatment. Every cycle the bicyclist(s) were shown a green indication before the vehicles were. The vehicles in the thru and shared thru/right-turn lanes were shown a red indication for $5 \mathrm{sec}$, before being shown a green indication. All three approaches (bike lane, vehicle thru lane, and vehicle thru/right-turn lane) ended at the same time using the same amber and red clearance times.

Results of the LBI simulations can be seen for vehicles in Table 5.1, Figure 5.1, and Figure 5.2 below. All delays for the LBI treatment's movements were statistically significant to $p=0.05$ The LBI showed uniform increased delay across all approaches. This is expected as the LBI impedes all vehicle traffic for $5 \mathrm{sec}$, which (conveniently) is the same amount of increased delay seen regardless of bicycle volume. 


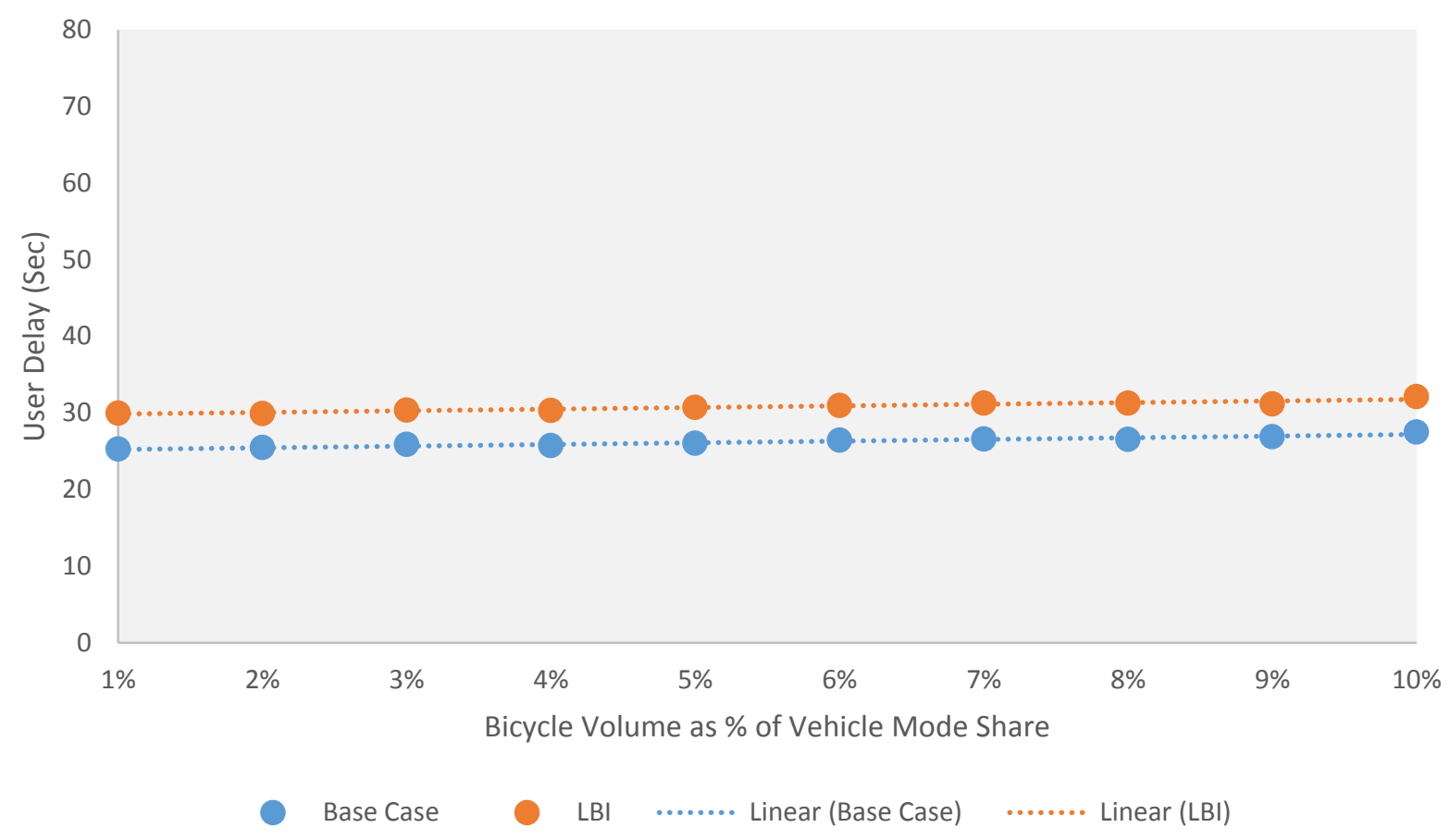

Figure 5.1: LBI vehicle thru movement delay results

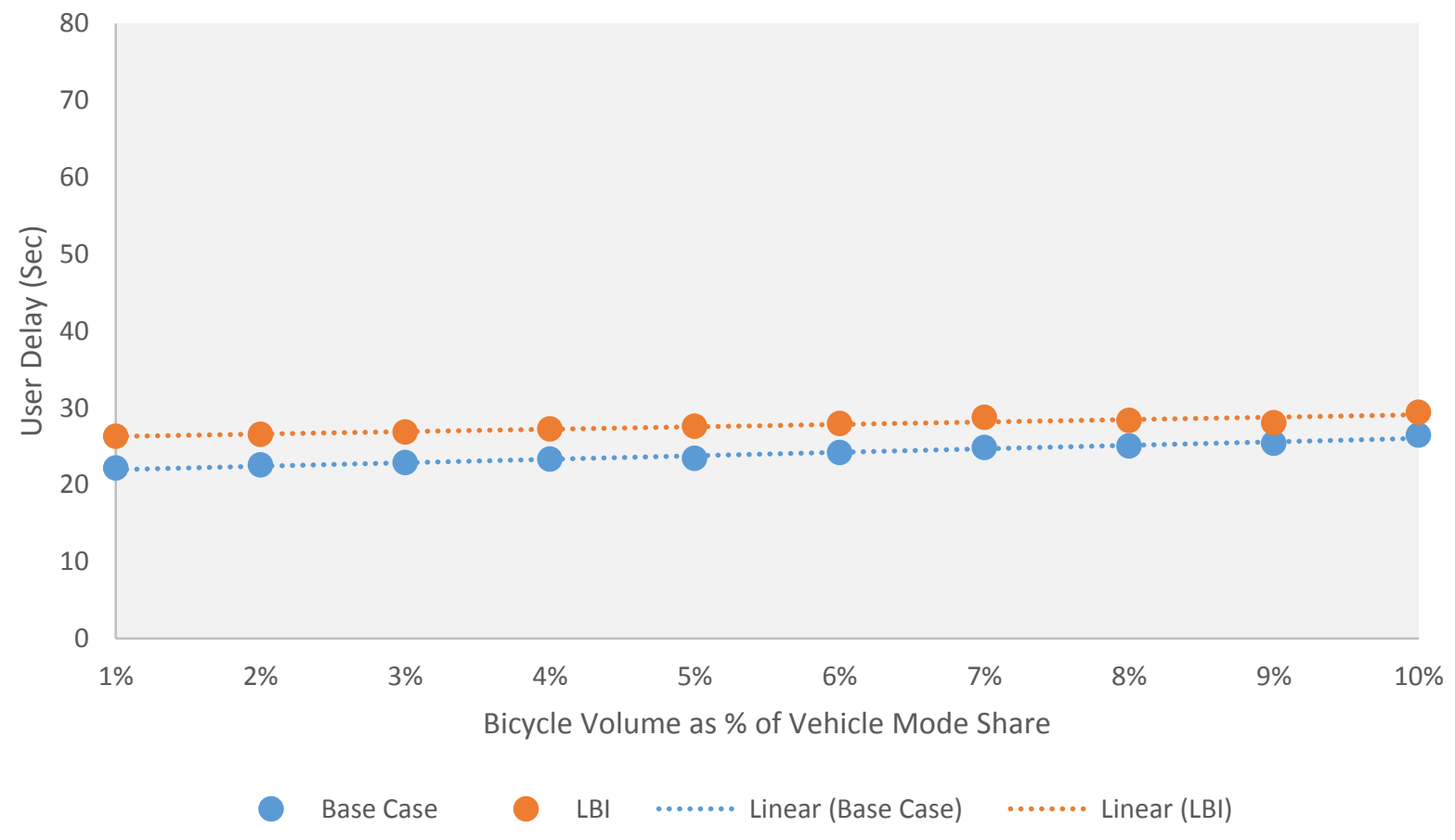

Figure 5.2: LBI vehicle right turn movement delay results 
Table 5.1: LBI (Phase 6) Vehicle Sensitivity Analysis Delay Results

\begin{tabular}{lccccccccccc}
\hline \multirow{2}{*}{ Movement and Case } & \multicolumn{8}{c}{ Bicycle Volume as Percent of Mode Share, Delay (secs) } \\
\cline { 3 - 11 } & $\mathbf{1 \%}$ & $\mathbf{2 \%}$ & $\mathbf{3 \%}$ & $\mathbf{4 \%}$ & $\mathbf{5 \%}$ & $\mathbf{6 \%}$ & $\mathbf{7 \%}$ & $\mathbf{8 \%}$ & $\mathbf{9 \%}$ & $\mathbf{1 0 \%}$ \\
\hline \multirow{3}{*}{ Thru } & Base Case Delay & 25.3 & 25.5 & 25.9 & 25.8 & 26.0 & 26.4 & 26.6 & 26.6 & 26.9 & 27.5 \\
& LBI Delay & $\mathbf{3 0 . 0}$ & $\mathbf{2 9 . 9}$ & $\mathbf{3 0 . 4}$ & $\mathbf{3 0 . 3}$ & $\mathbf{3 0 . 7}$ & $\mathbf{3 1 . 0}$ & $\mathbf{3 1 . 3}$ & $\mathbf{3 1 . 3}$ & $\mathbf{3 1 . 2}$ & $\mathbf{3 2 . 1}$ \\
& \% Difference & $19 \%$ & $17 \%$ & $17 \%$ & $18 \%$ & $18 \%$ & $17 \%$ & $18 \%$ & $18 \%$ & $16 \%$ & $17 \%$ \\
\hline \multirow{2}{*}{ Right } & Base Case Delay & 22.2 & 22.6 & 22.9 & 23.3 & 23.4 & 24.2 & 24.8 & 25 & 25.4 & 26.5 \\
Turn & LBI Delay & $\mathbf{2 6 . 3}$ & $\mathbf{2 6 . 6}$ & $\mathbf{2 6 . 9}$ & $\mathbf{2 7 . 2}$ & $\mathbf{2 7 . 6}$ & $\mathbf{2 7 . 9}$ & $\mathbf{2 8 . 8}$ & $\mathbf{2 8 . 4}$ & $\mathbf{2 8 . 1}$ & $\mathbf{2 9 . 4}$ \\
& \% Difference & $19 \%$ & $18 \%$ & $18 \%$ & $17 \%$ & $18 \%$ & $16 \%$ & $16 \%$ & $13 \%$ & $11 \%$ & $11 \%$ \\
\hline
\end{tabular}

Bolded cells are statistically significant to the $\mathbf{9 5 \%}$ CI.

Bicycle delay values did not follow this trend (Table 5.2, Figure 5.1, and Figure 5.2), instead showing little overall change, although none of the results were statistically significant. Indeed the change in percent difference seen in Table 5.2 shows how little bicycle delay appears to be affected by the LBI treatment; not only were results of the percent difference between the base case and the LBI low, but none of the results were statistically significant.

Table 5.2: LBI (Phase 6) Bicycle Sensitivity Analysis Delay Results

\begin{tabular}{llccccccccccc}
\hline \multirow{2}{*}{ Movement and Case } & \multicolumn{8}{c}{ Bicycle Volume as Percent of Mode Share, Delay (secs) } \\
\cline { 3 - 12 } & $\mathbf{1 \%}$ & $\mathbf{2 \%}$ & $\mathbf{3 \%}$ & $\mathbf{4 \%}$ & $\mathbf{5 \%}$ & $\mathbf{6 \%}$ & $\mathbf{7 \%}$ & $\mathbf{8 \%}$ & $\mathbf{9 \%}$ & $\mathbf{1 0 \%}$ \\
\hline \multirow{2}{*}{ Thru } & Base Case Delay & 17.8 & 18.8 & 17.3 & 18.1 & 20.5 & 20.1 & 21.1 & 23.6 & 25.1 & 22.5 \\
& LBI Delay & 18.1 & 18.7 & 16.9 & 18.7 & 21.0 & 20.4 & 20.9 & 24.3 & 25.1 & 22.6 & 0 \\
& \% Difference & $2 \%$ & $-1 \%$ & $-2 \%$ & $4 \%$ & $2 \%$ & $1 \%$ & $-1 \%$ & $3 \%$ & $0 \%$ & $1 \%$ \\
\hline \multirow{2}{*}{ Right } & Base Case Delay & 2.2 & 7.0 & 5.9 & 8.6 & 10.4 & 10.4 & 12.1 & 13.2 & 16.4 & 13.1 \\
Turn & LBI Delay & 2.2 & 7.1 & 6.0 & 8.2 & 10.8 & 9.9 & 11.7 & 14.2 & 16.4 & 14.3 \\
& \% Difference & $0 \%$ & $2 \%$ & $1 \%$ & $-5 \%$ & $4 \%$ & $-4 \%$ & $-4 \%$ & $8 \%$ & $0 \%$ & $5 \%$ \\
\hline
\end{tabular}

Bolded cells are statistically significant to the $\mathbf{9 5 \%} \mathrm{CI}$.

\subsubsection{Split LBI (Eastbound)}

The Eastbound approach of the model (phase 2) used the Split LBI treatment. Every cycle the bicyclist(s) and the vehicle thru movements were shown a green indication before the right turning vehicles were. The vehicles in the right turn lane were shown a red indication for $5 \mathrm{sec}$, before being shown a green indication. All three approaches (bike lane, vehicle thru lane, and vehicle right turn lane) ended at the same time using the same amber and red clearance times. Table 5.3, Figure 5.3, and Figure 5.4 show the vehicle delay results for the base case compared with the Split LBI treatment. 


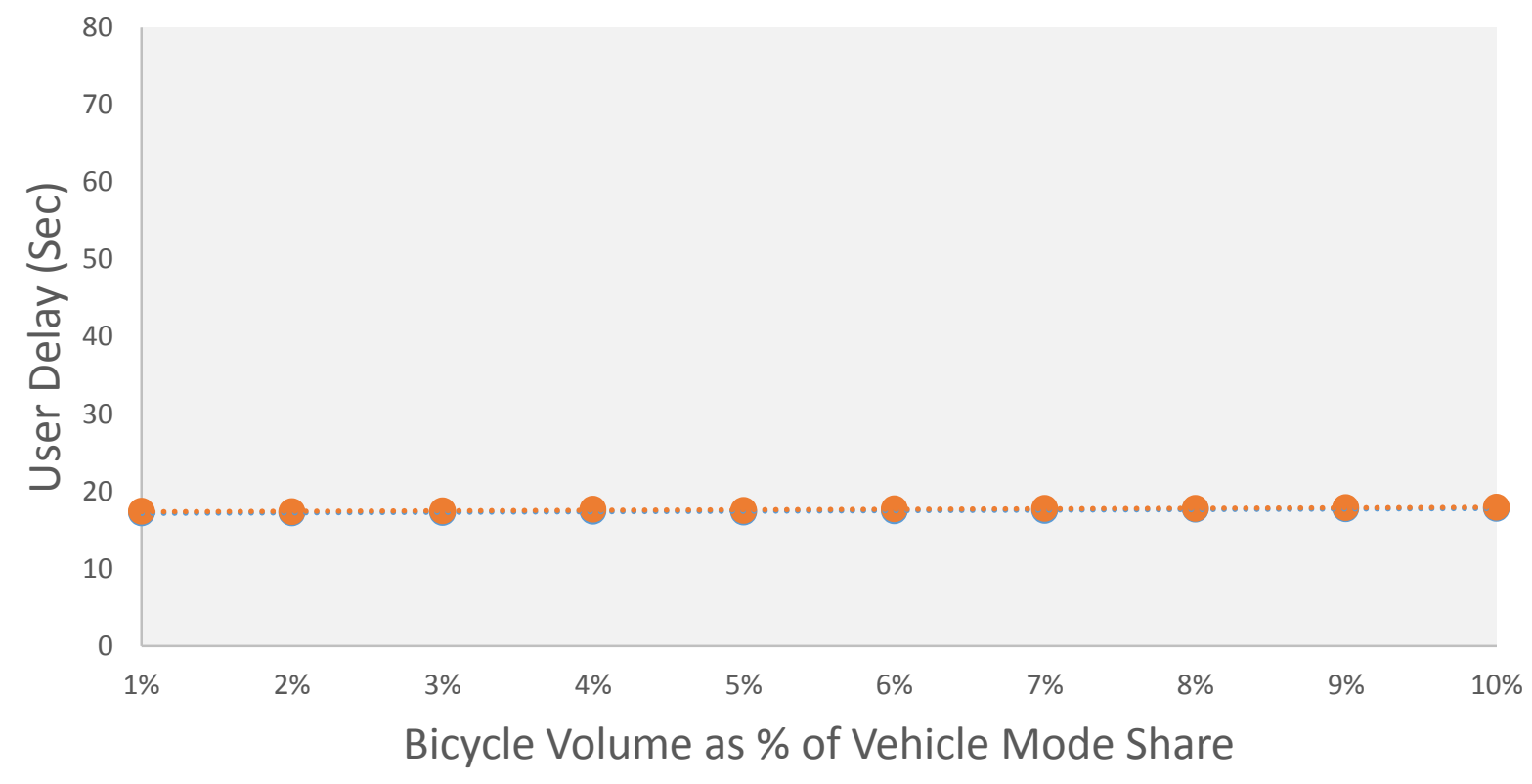

- Base Case Split LBI $\quad \cdots . . . .$. Linear (Base Case) $\quad \cdots . . . .$. Linear (Split LBI)

Figure 5.3: Split LBI Vehicle Thru Movement Delay Results

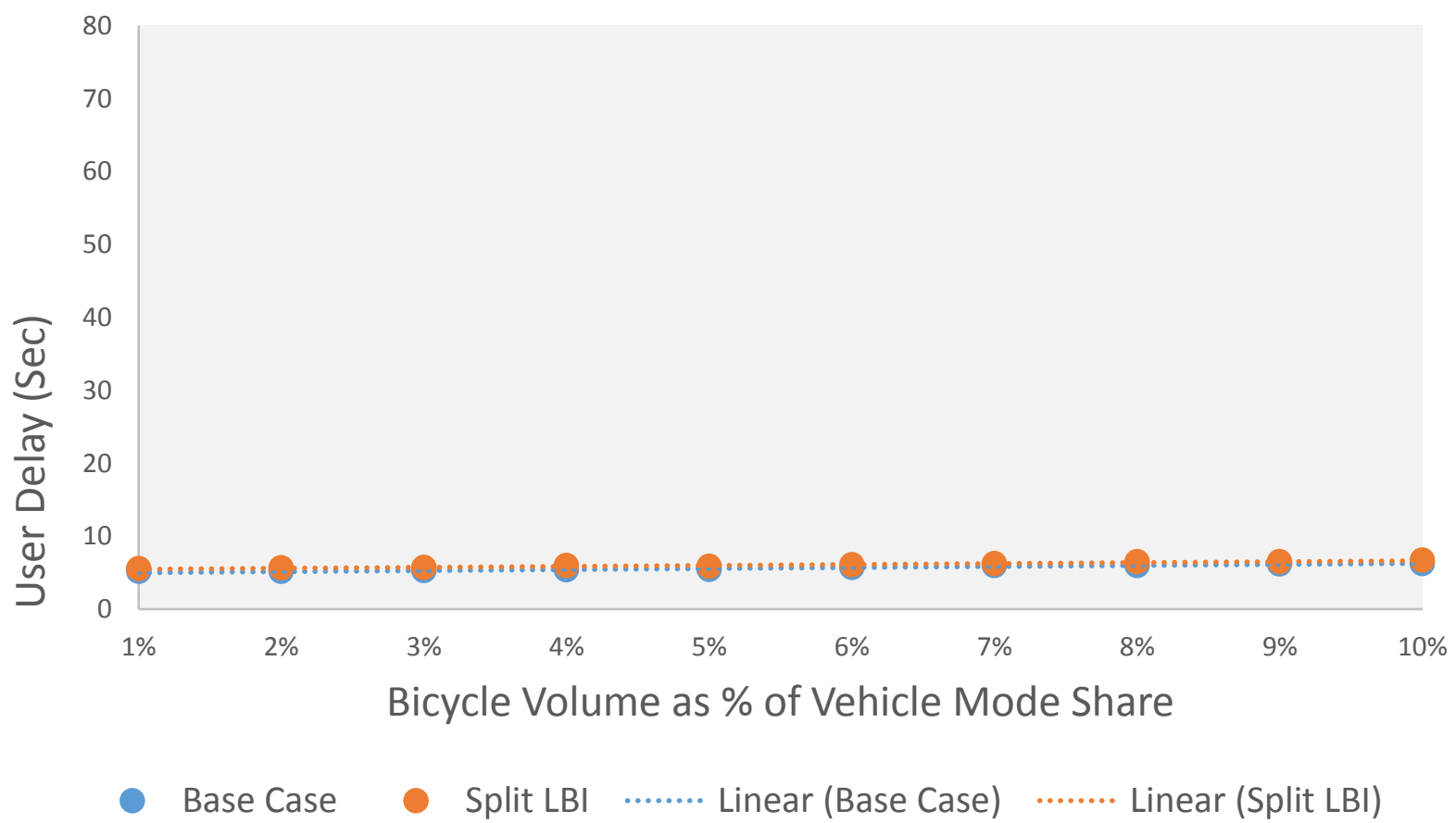

Figure 5.4: Split LBI Vehicle Right Turn Movement Delay Results 
Table 5.3: Split LBI (Phase 2) Vehicle Sensitivity Analysis Delay Results

\begin{tabular}{lccccccccccc}
\hline \multirow{2}{*}{ Movement and Case } & \multicolumn{8}{c}{ Bicycle Volume as Percent of Mode Share, Delay (secs) } \\
\cline { 3 - 11 } & & $\mathbf{1 \%}$ & $\mathbf{2 \%}$ & $\mathbf{3 \%}$ & $\mathbf{4 \%}$ & $\mathbf{5 \%}$ & $\mathbf{6 \%}$ & $\mathbf{7 \%}$ & $\mathbf{8 \%}$ & $\mathbf{9 \%}$ & $\mathbf{1 0 \%}$ \\
\hline \multirow{3}{*}{ Thru } & Base Case Delay & 17.2 & 17.2 & 17.3 & 17.4 & 17.3 & 17.5 & 17.5 & 17.7 & 17.7 & 17.8 \\
& Split LBI Delay & $\mathbf{1 7 . 4}$ & 17.4 & 17.5 & $\mathbf{1 7 . 7}$ & 17.5 & $\mathbf{1 7 . 8}$ & $\mathbf{1 7 . 8}$ & 17.8 & 17.9 & 17.9 \\
& \% Difference & $1 \%$ & $1 \%$ & $1 \%$ & $2 \%$ & $1 \%$ & $2 \%$ & $2 \%$ & $1 \%$ & $1 \%$ & $1 \%$ \\
\hline \multirow{2}{*}{ Right } & Base Case Delay & 5.15 & 5.18 & 5.26 & 5.39 & 5.43 & 5.71 & 5.97 & 5.99 & 6.18 & 6.23 \\
\multirow{2}{*}{ Turn } & Split LBI Delay & $\mathbf{5 . 5 5}$ & $\mathbf{5 . 6 6}$ & $\mathbf{5 . 7 1}$ & $\mathbf{5 . 9 9}$ & $\mathbf{5 . 8 4}$ & $\mathbf{6 . 7}$ & $\mathbf{6 . 2 2}$ & $\mathbf{6 . 4 9}$ & $\mathbf{6 . 4 9}$ & $\mathbf{6 . 7}$ \\
& \% Difference & $8 \%$ & $9 \%$ & $9 \%$ & $11 \%$ & $8 \%$ & $6 \%$ & $4 \%$ & $8 \%$ & $5 \%$ & $8 \%$ \\
\hline
\end{tabular}

Bolded cells are statistically significant to the $\mathbf{9 5 \%}$ CI.

The difference in delay caused by the Split LBI was nearly negligible for the unaffected thru movements (which showed little statistical significance), and was relatively low ( $<1 \mathrm{sec})$ for the right turns (but highly statistically significant). Both of these results are expected; the thru movements are not impeded by the Split LBI and would therefore be expected to show little change; the right turns are impeded for $5 \mathrm{sec}$ in the entire cycle length, minimizing the magnitude of the impact.

The effects on bicycle traffic was also studied with the results being listed in Table 5.4, Figure 5.5, and Figure 5.6 below. Bicycle results for the thru movements appeared to show minor changes in delay, but with only a few runs being statistically significant. The increases at the higher bicycle volumes is likely the result of queuing delays caused by the increasing number of bicyclists, which at higher bicycle volumes begin to experience platoon dispersion delays the same way a vehicle would. 


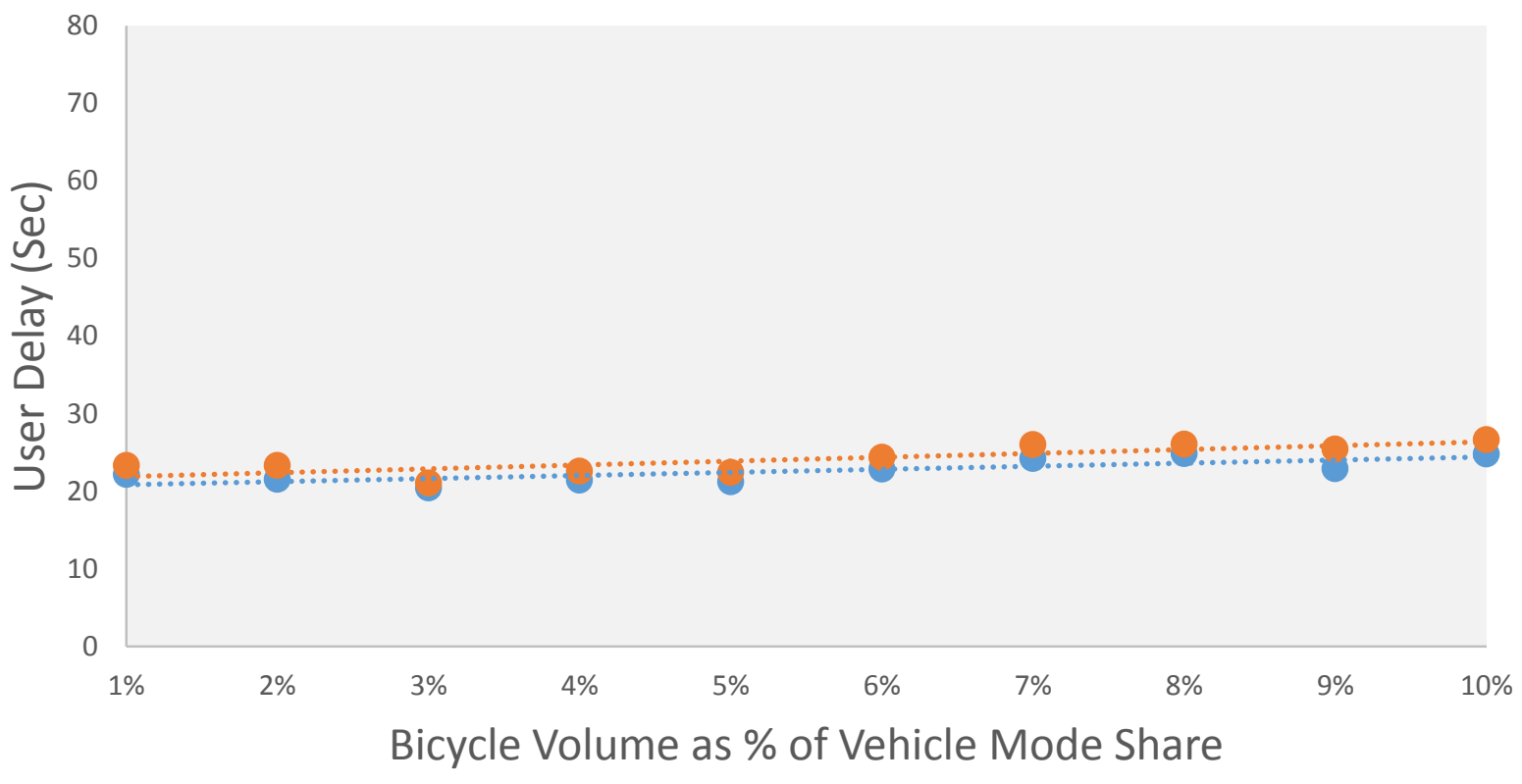

- Base Case Split LBI …..... Linear (Base Case) …..... Linear (Split LBI)

Figure 5.5: Split LBI Bicycle Thru Movement Delay Results

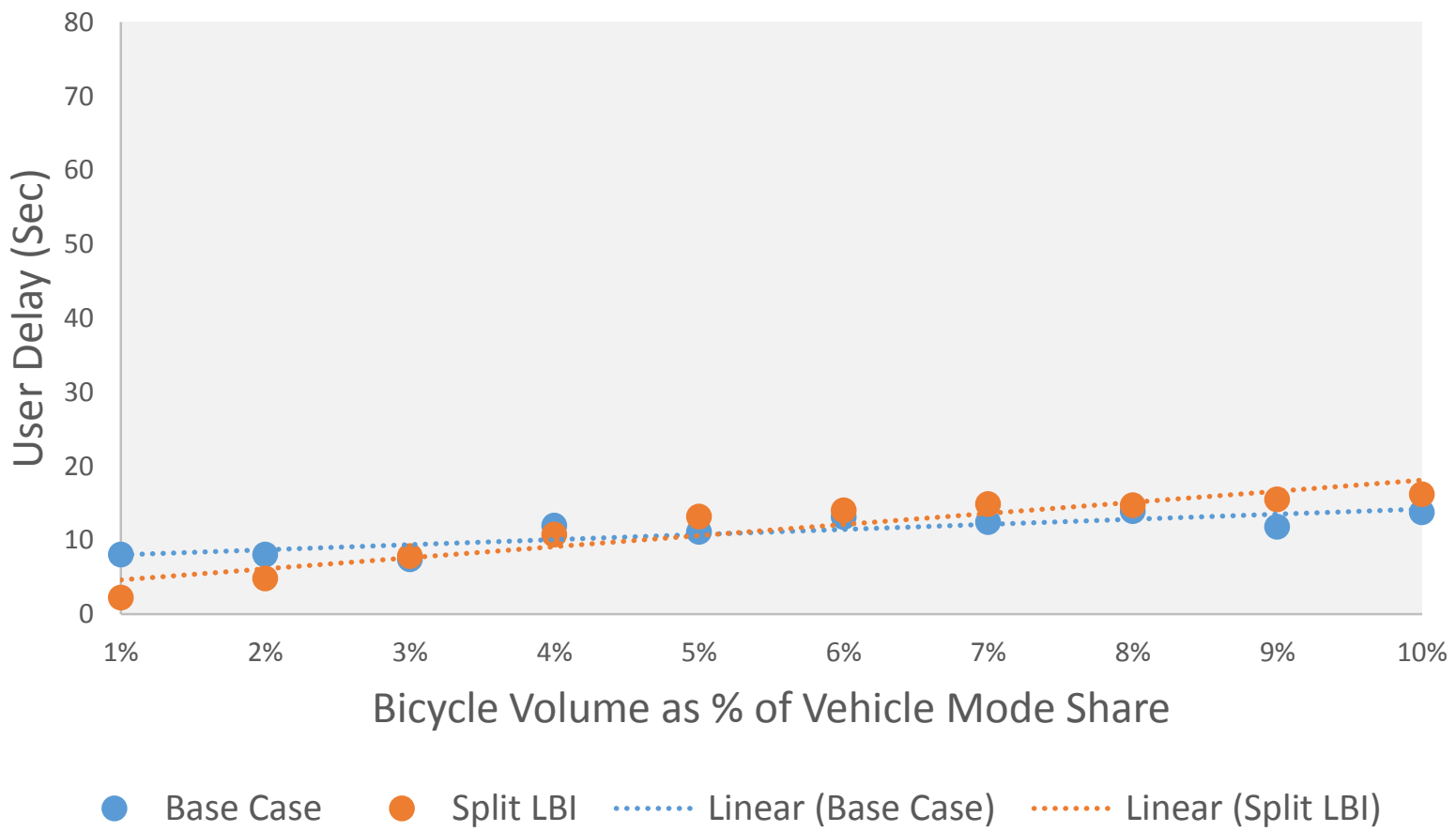

Figure 5.6: Split LBI Bicycle Right Turn Movement Delay Results 
Table 5.4: Split LBI (Phase 2) Bicycle Sensitivity Analysis Delay Results

\begin{tabular}{llcccccccccc}
\hline & & \multicolumn{7}{c}{ Bicycle Volume as Percent of Mode Share, Delay (secs) } \\
\cline { 3 - 11 } & & $\mathbf{1 \%}$ & $\mathbf{2 \%}$ & $\mathbf{3 \%}$ & $\mathbf{4 \%}$ & $\mathbf{5 \%}$ & $\mathbf{6 \%}$ & $\mathbf{7 \%}$ & $\mathbf{8 \%}$ & $\mathbf{9 \%}$ & $\mathbf{1 0 \%}$ \\
\hline \multirow{3}{*}{ Thru } & Base Case Delay & 22.2 & 21.5 & 20.4 & 21.4 & 21.2 & 22.8 & 24.2 & 24.9 & 22.9 & 24.8 \\
& Split LBI Delay & 23.3 & 23.3 & 21.1 & 22.6 & 22.4 & 24.4 & $\mathbf{2 6 . 0}$ & 26.1 & $\mathbf{2 5 . 4}$ & $\mathbf{2 6 . 6}$ \\
& \% Difference & $5 \%$ & $8 \%$ & $3 \%$ & $5 \%$ & $6 \%$ & $7 \%$ & $7 \%$ & $5 \%$ & $11 \%$ & $7 \%$ \\
\hline \multirow{2}{*}{ Right } & Base Case Delay & 8.0 & 8.0 & 7.4 & 11.9 & 11.1 & 13.1 & 12.4 & 13.9 & 11.8 & 13.7 \\
Turn & Split LBI Delay & 2.2 & 4.8 & 7.8 & 10.7 & 13.2 & 14.0 & 14.9 & 14.7 & $\mathbf{1 5 . 5}$ & 16.2 \\
& \% Difference & $-72 \%$ & $-40 \%$ & $6 \%$ & $-10 \%$ & $19 \%$ & $7 \%$ & $20 \%$ & $6 \%$ & $32 \%$ & $18 \%$ \\
\hline
\end{tabular}

Bolded cells are statistically significant to the $\mathbf{9 5 \%} \mathbf{C I}$.

Right turning movements showed an odd trend where the Split LBI began as having substantially less delay that the base case, but increased quickly until the treatment delay surpassed the base case delay (although only the $9 \%$ bike volume scenario is significant). These results are almost certainly due to bike queuing issues. The more bicycles in the system the longer they will wait in the queue, as there is no dedicated right turn lane for bicyclists. At lower volumes the bicyclists

are less likely to encounter a queue and would have a better chance of making their turns without waiting.

\subsubsection{Minor Approach Phases}

The minor phases of the intersection were not altered in anyway, including the low bike volumes obtained from Quality Counts. Bicycle results were excluded due to very low volumes, which riddled the results with small sample errors. Since the LBI and Split LBI treatments do not directly affect the minor approaches they would be expected to experience very little impact from the implementation of the treatments. Table 5.5 shows the percent difference in delay due to the treatments.

Table 5.5: Minor Phases (Phases 4 and 8) Vehicle and Bicycle Sensitivity Analysis Delay Results

\begin{tabular}{|c|c|c|c|c|c|c|c|c|c|c|c|}
\hline \multirow{2}{*}{\multicolumn{2}{|c|}{ Movement and Case }} & \multicolumn{10}{|c|}{ Bicycle Volume as Percent of Mode Share, Delay as Percent Difference } \\
\hline & & $1 \%$ & $2 \%$ & $3 \%$ & $4 \%$ & $5 \%$ & $6 \%$ & $7 \%$ & $\mathbf{8 \%}$ & $9 \%$ & $10 \%$ \\
\hline \multirow{3}{*}{ Southbound } & Veh Thru & $0 \%$ & $0 \%$ & $0 \%$ & $0 \%$ & $0 \%$ & $1 \%$ & $0 \%$ & $0 \%$ & $0 \%$ & $0 \%$ \\
\hline & Veh Right Turn & $0 \%$ & $0 \%$ & $-2 \%$ & $2 \%$ & $-3 \%$ & $-1 \%$ & $-1 \%$ & $-2 \%$ & $-1 \%$ & $1 \%$ \\
\hline & Bicycle Thru & $0 \%$ & $0 \%$ & $0 \%$ & $0 \%$ & $0 \%$ & $0 \%$ & $0 \%$ & $0 \%$ & $0 \%$ & $0 \%$ \\
\hline \multirow{3}{*}{ Northbound } & Veh Thru & $0 \%$ & $0 \%$ & $0 \%$ & $0 \%$ & $0 \%$ & $1 \%$ & $1 \%$ & $1 \%$ & $1 \%$ & $1 \%$ \\
\hline & Veh Right Turn & $0 \%$ & $0 \%$ & $1 \%$ & $-1 \%$ & $1 \%$ & $1 \%$ & $2 \%$ & $1 \%$ & $0 \%$ & $3 \%$ \\
\hline & Bicycle Thru & $-15 \%$ & $-15 \%$ & $-16 \%$ & $-15 \%$ & $-15 \%$ & $-15 \%$ & $-15 \%$ & $-15 \%$ & $-15 \%$ & $-15 \%$ \\
\hline
\end{tabular}

Bolded cells are statistically significant to the $\mathbf{9 5 \%}$ CI.

The results indicate little to no change at the minor approaches, with only a few being statistically significant. There are however two notable exceptions: southbound right turn and 
northbound bicycle thru. Southbound right turn saw slight decreases in delay at the higher bike volume percentages, which is explained by the LBI; the same $5 \mathrm{sec}$ that impede the westbound thru movements also gives the southbound right turn an additional $5 \mathrm{sec}$ in which to make a turn.

The drop in delay seen in the northbound bicycle thru movement is the result of small sample error. Specifically, the 9th run of each 10 run set in the base case scenario; the two bicyclists who used the approach had delay values of $74.56 \mathrm{sec}$, compared to the $25.39 \mathrm{sec}$ of delay seen in the 9th run of the 10 run set for the LBI scenarios. Essentially the same bicyclist arrived at beginning of the red indication on the northbound approach for each of the 9th runs. The bicyclist in question entered the model at the same time each run, regardless of the treatment type being implemented. Since the LBI scenario had different timing splits it would be expected that the state of an individual indication would be different for the base case and the LBI at the same time-step in the simulation. Meaning that the bicyclist would enter at time $\mathrm{X}$ and hit the early stages of the red indication in the base case, and then arrive at time $\mathrm{X}$ in the LBI and receive a different indication, which in turn resulted in a decrease in delay. Additionally, only two bicyclists used the approach, and the small sample size disproportionately affects the results.

\subsubsection{Left Turning Phases}

Left turning phases of the intersection were not altered. Since the LBI and Split LBI treatments do not directly affect the left turn approaches it would be expected that they would experience very little impact from the implementation of the treatments. Table 5.6 shows the percent difference in delay results due to the treatments.

Table 5.6: Left Turn Vehicle Sensitivity Analysis Delay Results

\begin{tabular}{lcccccccccc}
\hline \multirow{2}{*}{ Movement } & \multicolumn{8}{c}{ Bicycle Volume as Percent of Mode Share, Delay as Percent Difference } \\
\cline { 2 - 10 } & $\mathbf{1 \%}$ & $\mathbf{2 \%}$ & $\mathbf{3 \%}$ & $\mathbf{4 \%}$ & $\mathbf{5 \%}$ & $\mathbf{6 \%}$ & $\mathbf{7 \%}$ & $\mathbf{8 \%}$ & $\mathbf{9 \%}$ & $\mathbf{1 0 \%}$ \\
\hline WB LT & $1 \%$ & $1 \%$ & $1 \%$ & $0 \%$ & $3 \%$ & $2 \%$ & $1 \%$ & $2 \%$ & $1 \%$ & $1 \%$ \\
NB LT & $3 \%$ & $2 \%$ & $2 \%$ & $2 \%$ & $3 \%$ & $2 \%$ & $2 \%$ & $2 \%$ & $3 \%$ & $\mathbf{3 \%}$ \\
EB LT & $1 \%$ & $1 \%$ & $1 \%$ & $1 \%$ & $\mathbf{2 \%}$ & $0 \%$ & $\mathbf{3 \%}$ & $1 \%$ & $1 \%$ & $1 \%$ \\
SB LT & $1 \%$ & $0 \%$ & $1 \%$ & $1 \%$ & $1 \%$ & $1 \%$ & $1 \%$ & $2 \%$ & $2 \%$ & $2 \%$ \\
\hline
\end{tabular}

Bolded cells are statistically significant to the $\mathbf{9 5 \%}$ CI.

The largest percent difference in delay is $3 \%$, which represents $1.5 \mathrm{sec}$ of additional delay. The remaining phases show little to no change suggesting the LBI treatments had little effect on left turns. However only a few of the results were statistically significant. Any vehicle delay increase in the left turns is likely the result of slight variations in vehicle arrivals.

\subsubsection{Pedestrian Movements}

All pedestrian movements, phases 1 - 8, were included in the Vissim model. Pedestrian movements were not altered by the LBI treatments. The LBI and the corresponding pedestrian 
movement began at the same time, with the WALK and the LBI/Split LBI green turning on simultaneously. The percent difference in delay for each movement is shown in Table 5.7, with statistical significance of the means being represented within these results.

Table 5.7: Pedestrian Sensitivity Analysis Delay Results

\begin{tabular}{lcccccccccc}
\hline \multirow{2}{*}{$\begin{array}{c}\text { Crosswalk and } \\
\text { Direction }\end{array}$} & \multicolumn{8}{c}{ Bicycle Volume as Percent of Mode Share, Delay as Percent Difference } \\
\cline { 2 - 11 } & $\mathbf{1 \%}$ & $\mathbf{2 \%}$ & $\mathbf{3 \%}$ & $\mathbf{4 \%}$ & $\mathbf{5 \%}$ & $\mathbf{6 \%}$ & $\mathbf{7 \%}$ & $\mathbf{8 \%}$ & $\mathbf{9 \%}$ & $\mathbf{1 0 \%}$ \\
\hline Southside EB & $0 \%$ & $0 \%$ & $0 \%$ & $0 \%$ & $0 \%$ & $0 \%$ & $0 \%$ & $0 \%$ & $0 \%$ & $0 \%$ \\
Southside WB & $0 \%$ & $0 \%$ & $1 \%$ & $1 \%$ & $0 \%$ & $1 \%$ & $1 \%$ & $0 \%$ & $0 \%$ & $0 \%$ \\
\hline Westside NB & $0 \%$ & $\mathbf{0 \%}$ & $0 \%$ & $0 \%$ & $0 \%$ & $0 \%$ & $0 \%$ & $0 \%$ & $0 \%$ & $0 \%$ \\
Westside SB & $0 \%$ & $0 \%$ & $0 \%$ & $0 \%$ & $0 \%$ & $0 \%$ & $0 \%$ & $0 \%$ & $0 \%$ & $0 \%$ \\
\hline Northside EB & $\mathbf{1 \%}$ & $1 \%$ & $-1 \%$ & $1 \%$ & $0 \%$ & $0 \%$ & $0 \%$ & $\mathbf{1 \%}$ & $1 \%$ & $1 \%$ \\
Northside WB & $0 \%$ & $\mathbf{2 \%}$ & $1 \%$ & $2 \%$ & $1 \%$ & $1 \%$ & $1 \%$ & $\mathbf{2 \%}$ & $\mathbf{3 \%}$ & $\mathbf{3 \%}$ \\
\hline Eastside NB & $0 \%$ & $0 \%$ & $0 \%$ & $0 \%$ & $0 \%$ & $0 \%$ & $0 \%$ & $0 \%$ & $0 \%$ & $0 \%$ \\
Eastside SB & $0 \%$ & $0 \%$ & $0 \%$ & $0 \%$ & $0 \%$ & $0 \%$ & $0 \%$ & $0 \%$ & $0 \%$ & $-1 \%$ \\
\hline Botded
\end{tabular}

Bolded cells are statistically significant to the $\mathbf{9 5 \%}$ CI.

The results show how little the LBI treatments affected the pedestrian movements. The only pedestrian phase that shows any meaningful change is phase 6 (the north side of the intersection), especially the westbound pedestrians. The largest percent difference is 3\% on "Northside WB", which represents a $0.74 \mathrm{sec}$ difference in additional delay. Phase 6 pedestrian movements had the lowest volume of pedestrians for any of the four pedestrian approaches, with a total of 14 pedestrians each direction (14 eastbound and 14 westbound for a total of 28 pedestrians) for the entire hour. Many of the runs saw only one pedestrian use the approach in a cycle. Almost all runs that were statistically significant were seen on results from North side (pedestrian phase 6), the LBI treatment. The increases seen are likely the result of small sample errors, or what could be deemed normal fluctuations.

\subsection{Exclusive Bicycle Phase (EBP)}

The final treatment examined was the Exclusive Bicycle Phase (EBP). In this scheme all traffic is held in order to allow select bicycle movements unrestricted access to the intersection (similar to an Exclusive Pedestrian Phase). In the version applied to this model only eastbound (phase 2) and westbound (phase 6) bicycles were given the EBP, while the minor northbound (phase 8) and southbound (phase 4) were not.

Results from the simulation are shown in three tables below; Table 5.8 shows the vehicle delay results, Table 5.9 shows the bicycle delay results, and Table 5.10 shows the pedestrian delay results. Each will be discussed in turn. 
Table 5.8: Exclusive Bicycle Phase (Phases 2 and 6) Vehicle Delay Results

\begin{tabular}{cccc}
\hline Movement & Base Case (sec) & EBP $(\mathbf{s e c})$ & \% Difference \\
\hline EB TH & 17.2 & $\mathbf{2 1 . 6 8}$ & $26 \%$ \\
EB RT & 5.15 & $\mathbf{5 . 5 2}$ & $7 \%$ \\
EB LT & 62.5 & $\mathbf{7 4 . 5 2}$ & $19 \%$ \\
\hline WB TH & 25.28 & $\mathbf{2 1 . 2 3}$ & $-16 \%$ \\
WB RT & 22.16 & $\mathbf{1 9 . 2 6}$ & $-13 \%$ \\
WB LT & 52.23 & $\mathbf{5 6 . 2 5}$ & $8 \%$ \\
\hline SB TH & 34.12 & 35.15 & $3 \%$ \\
SB RT & 6.12 & 6.7 & $-1 \%$ \\
SB LT & 54.81 & 65.81 & $20 \%$ \\
\hline NB TH & 37.1 & 37.64 & $1 \%$ \\
NB RT & 7.4 & 7.77 & $5 \%$ \\
NB LT & 53.1 & $\mathbf{5 4 . 7 4}$ & $3 \%$ \\
\hline
\end{tabular}

Bolded cells are statistically significant to the $\mathbf{9 5 \%} \mathbf{C I}$.

Vehicle delay in Table 5.8 showed mixed results, with the eastbound movements experiencing substantial increases, which were also statistically significant. The westbound thru and right turn movements showed decreases in delay and were statistically significant. Southbound and Northbound showed little change and were not statistically significant, with the exception of northbound left turn which saw a minor increase in delay. Left turns saw increases in delay, with three of the four being statistically significant.

These increases and decreases appear to be the result of an unintentional favoring of the westbound (phase 6) approach in the coordination scheme. The Synchro time-space diagrams show not only the travel paths of vehicles but also delay estimates. The diagrams showed an increase in delay for the eastbound (phase 2) approach (increasing from $15 \mathrm{sec}$ in the base case to $18 \mathrm{sec}$ in the EBP), while the westbound approach (phase 6) showed a decrease in delay (from 29 sec in the base case to $24 \mathrm{sec}$ in the EBP). This would explain the odd results seen in Table 5.8. 
Table 5.9: Exclusive Bicycle Phase (Phases 2 and 6) Bicycle Delay Results

\begin{tabular}{cccc}
\hline Movement & Base Case (sec) & EBP $(\mathbf{s e c})$ & \% Diff \\
\hline EB TH & 22.17 & $\mathbf{4 5 . 6 3}$ & $16 \%$ \\
EB RT & 8.2 & 6.1 & $-25 \%$ \\
EB LT & 42.65 & $\mathbf{8 5 . 4 6}$ & $100 \%$ \\
\hline WB TH & 17.75 & $\mathbf{4 4 . 6 5}$ & $152 \%$ \\
WB RT & 2.15 & 14.29 & $565 \%$ \\
WB LT & 29.29 & 40.77 & $39 \%$ \\
\hline SB TH & 33.29 & $\mathbf{3 0 . 6 2}$ & $-8 \%$ \\
SB RT & 0 & 0 & - \\
SB LT & 0 & 0 & - \\
\hline NB TH & 35.36 & 25.72 & $-27 \%$ \\
NB RT & 3.22 & 3.26 & $1 \%$ \\
NB LT & 54.62 & $\mathbf{5 0 . 6 6}$ & $-7 \%$ \\
\hline
\end{tabular}

Bolded cells are statistically significant to the $\mathbf{9 5 \%} \boldsymbol{C I}$.

Table 5.9 bicycle delay results for eastbound bicycles showed an increase in the thru and left turn movements, but a decrease in the right turns, with only the former being statistically significant. Westbound bicycles showed a statistically significant increase in delay for the thru movement. Southbound bicycles showed a statistically significant decrease in delay for the thru movement, with zero values for the right and left turns (because no bicyclists used them, see section 4.2 above). Northbound bicycles showed a statistically significant decrease in bicyclist delay for the left turn movement.

Since the EBP only allows bicyclists to proceed at end of the signal cycle (during phase 9 only), increases in delay would be expected, and the results seem to demonstrate this increase.

Although caution should be used in that the eastbound and westbound turning values are the result of only a few bicyclists, strongly suggesting small sample influence.

The drop in delay seen on the minor northbound and southbound right turn movements is probably the result of increased opportunity to make unencumbered right turns; conflicting eastbound or westbound bicycles are stopped at the EBP for the majority of the signal cycle. 
Table 5.10: Exclusive Bicycle Phase (Phases 2 and 6) Pedestrian Delay Results

\begin{tabular}{cccc}
\hline $\begin{array}{c}\text { Crosswalk Direction and } \\
\text { Direction }\end{array}$ & Base Case (sec) & EBP $(\mathbf{s e c})$ & \% Diff \\
\hline Southside EB & 29.8 & $\mathbf{3 3 . 5 9}$ & $16 \%$ \\
Southside WB & 27.75 & $\mathbf{3 3 . 8 6}$ & $22 \%$ \\
Westside SB & 50.73 & 51.19 & $1 \%$ \\
Westside NB & 50.37 & 51.8 & $3 \%$ \\
Northside EB & 30.77 & $\mathbf{3 5 . 9 5}$ & $17 \%$ \\
Northside WB & 26.67 & $\mathbf{3 1 . 1 6}$ & $17 \%$ \\
Eastside NB & 54.1 & 52.4 & $-4 \%$ \\
Eastside SB & 52.3 & 50.1 & $-4 \%$ \\
\hline
\end{tabular}

Bolded cells are statistically significant to the $\mathbf{9 5 \%}$ CI.

Pedestrian delay results seen in Table 5.10 showed statistical significance for only the westbound and eastbound movements, both of which saw pronounced increases in delay. This is expected as both phases, like the vehicle phases, were delayed by the EBP. Southbound and northbound saw small, statistically non-significant, changes in delay values, both were not directly affected by the EBP.

The EBP pedestrian phases saw a near uniform $\pm 5 \mathrm{sec}$ increase in delay, while the minor approaches saw little to no change, which could be the result of cycle length reallocation. Since cycle length remained at $110 \mathrm{sec}$, with the EBP taking up approximately $16 \mathrm{sec}$ of that. That difference in time was taken largely from phases 2 and 6 (south side and north side in Table 5.10). This had the impact of reducing the available time for rest-in-walk, which in turn reduced the potential time that pedestrians had to access the intersection. This would be expected to increase pedestrian delay, which is what was observed. 


\section{CONCLUSIONS}

In order to understand the effects to intersection efficiency from the three bicycle specific treatments, microsimulation was used. The LBI, Split LBI, and EBP were modeled using Vissim, and each treatment was compared to a coordinated base case. The effects on user delay were recorded and analyzed.

Results for the LBI revealed little change in vehicle delay for the unaffected approaches (northbound and southbound), but a near uniform $5 \mathrm{sec}$ increase for the affected westbound approach. This $5 \mathrm{sec}$ is roughly the same as the $5 \mathrm{sec}$ from the LBI. Bicycle delay showed little change in delay, due largely to an unintentional favoring of the westbound approach in the coordination scheme.

Results for the Split LBI also revealed little change in vehicle delay for the unaffected approaches, including the unaffected thru movements on the Split LBI approach. There was a significant increase for the affected eastbound right turn movement, due to the treatment itself. The bicycles saw a slight increase in delay, which was likely the result of the eastbound approach being the un-favored approach in the coordination scheme. For both LBI and Split LBI pedestrian movements were all but unaffected.

The EBP vehicle delay results showed mixed outcomes; there was increased delay for the eastbound approach and decreased delay for the westbound. This difference is probably due to a favoring of the westbound approach in the coordination scheme. Minor phases were all but unaffected. EBP Bicycle results showed a general increase, which is probably due to the lack of signal time the EBP allocates to the bicycle movements $( \pm 10 \mathrm{sec})$. Pedestrian movements showed an increase in delay from the EBP, which was inferred to be the product of decreased pedestrian signal time as the result of cycle length reallocation (which was an outcome of the EBP). Minor pedestrian phases were unaffected.

\subsection{Areas of Further Research}

Bicyclist's behavior within Vissim was not as realistic as it could have been. Queuing and turning movements were two areas that were not particularly accurate. It was observed that during queueing bicyclists were spaced farther apart than they would be in real life. This could be remedied by adjusting the "Standstill Distance" for bicyclists; allowing for tighter "packingin" of bicyclists.

The turning movements for bicyclists had several issues: The left turning movements were coded in the model so that bicyclists used the intersections as a vehicle would. For left turns this means that the bicyclists would merge over two $35 \mathrm{mph}$ lanes of traffic to queue up with the left turning vehicles. In real life very few bicyclists would do this, preferring to either use the sidewalks and crossing as a pedestrian would, or to a make two-stage left turn. For the right turns many bicyclists would be expected to use the sidewalks to circumnavigate the intersection itself, either to avoid the potential right-hook conflict, or to avoid any queuing related delays.

If the experiment was to be performed again the recommendation would be use all of those options and code the bicyclists so that a certain percentage completed each turn type. For 
example, with the left turns: $10 \%$ of bicyclists would perform the turn as a vehicle would; $30 \%$ would complete a two-stage turn; and the remaining $60 \%$ would use the pedestrian facilities. These values are illustrative in nature, but video analysis would probably reveal the actual preferences of bicyclists.

A final area of exploration would be modifying the model to allow for bicyclists over taking each other, and queuing side by side. The model used in this research limited bicyclists to a single lane, which prevented both overtaking of slower bicyclists and also forced single file queueing. Neither of these assumptions are completely realistic, and the model could be adjusted to show this.

Safety of bicyclists is of paramount concern and is the reason that the treatments studied here were developed. All three treatments could be studied further to find the changes in conflicts that occur from their implementation. 


\section{REFERENCES}

Dill, Jennifer, and Nathan McNeil. 2013. "Four Types of Cyclists? Examination of Typology for Better Understanding of Bicycling Behavior and Potential." Transportation Research Record: Journal of the Transportation Research Board 129-138. doi:10.3141/2387-15.

Dill, Jennifer, Christopher Monsere, and Nathan McNeil. 2010. Evaluation of bike boxes at signalized intersections. Accident Analysis and Prevention, Elsevier, 126 - 134. doi:10.1016/j.aap.2010.10.030.

Hurwitz, David, Mafruhatul Jannat, Jennifer Warner, Christopher Monsere, and Ali Razmpa. 2015. Towards Effective Design Treatment for Right Turns at Intersections with Bicycle Traffic. Final, Washington, D.C.: Oregon Department of Transportation, 2 - 4. Accessed May 11, 2016. https://trid.trb.org/view.aspx?id=1375682.

MassDOT. 2016. Separated Bike Lane Planning and Design Guide. Massachusetts Department of Transportation: Highway Division.

ODOT. 2014. 2013 Oregon Crash Summary. Transportation Data Section Crash Analysis and Reporting Unit, Oregon Department of Transportation, Salem, OR: Oregon Department of Transportation, 4. https://www.oregon.gov/ODOT/TD/TDATA/car/docs/2013CrashSummaryBook.pdf.

ODOT. 2011. ODOT Vissim Protocol. Salem, OR: Oregon Department of Transportation.

Paulsen, Kirk, William Farley, Todd Mobley, Michael Ard, and Peter Koonce. 2016. "Analysis of Active Warning Sign to Address Potential Bicycle 'Right Hook' Conflict at Signalized intersections." Transportation Research Board 95th Annual Meeting.

Portland Bureau of Transportation. 2014. High Crash Corridors. August 5. Accessed September 16, 2015. http://www.portlandoregon.gov/transportation/article/439832.

Preusser, David F, William A Leaf, Karen B DeBartolo, and Richard D Blomberg. 1981. The Effect of Right-Turn-On-Red on Pedestrian and Bicyclist Accidents. USDOT: NHTSA.

PTV Group. 2014. Vissim 7 Manual. Karlsruhe, Germany: PTV Group. Accessed May 2016.

Pucher, John, Ralph Buehler, and Mark Seinen. 2011. "Bicycling renaissance in North America? An update and re-appraisal of cycling trends and policies." Transportation Research Part A: Policy and Practice 45 (6): 451-475. doi:10.1016/j.tra.2011.03.001.

Räsänen, Mikko, and Keikki Summala. 1998. "Attention and Expectation Problems in BicycleCar Collisions: An In-Depth Study." Accident Analysis and Prevention (Elsevier) 662.

Stokes, Bruce, Richard Wike, and Jill Carle. 2015. Global Concern about Climate Change, Broad Support for Limiting Emissions. Pew Research Center. Nov 5. Accessed May 9, 2016. http://www.pewglobal.org/2015/11/05/global-concern-about-climate-changebroad-support-for-limiting-emissions/.

Summala, Heikki, Mikko Räsänen, and Jukka Sievänen. 1996. "Bicycle Accidents and Drivers Visual Search at Left and Right Turns." Accident Analysis and Prevention (Elsevier).

U.S. Department of Transportation: Federal Highway Administration. n.d. Innovative Intersection Safety Improvement Strategies and Management Practices: A Domestic Scan. Accessed May 8, 2016. http://safety.fhwa.dot.gov/intersection/other_topics/fhwasa06016/chap_3.htm.

USDOT: NHTSA. 2012. Traffic Safety Facts, 2012 Data. Washington, D.C.: US Department of Transportation: National Highway Traffic Safety Administration. Accessed May 10, 2016. http://www-nrd.nhtsa.dot.gov/Pubs/812018.pdf.

Wachtel, Alan, and Diana Lewiston. 1995. "Risk Factors for Bicycle-Motor Vehicle Collisions at Intersections*." ITE Journal 30-35. 
